\title{
Theory and Techniques of Convergence of Topological Transformation Group Actions
}

\author{
Murtadha Jaber Sarray \\ WVU, mjsarray@mix.wvu.edu
}

Follow this and additional works at: https://researchrepository.wvu.edu/etd

Part of the Analysis Commons, and the Geometry and Topology Commons

\section{Recommended Citation}

Sarray, Murtadha Jaber, "Theory and Techniques of Convergence of Topological Transformation Group Actions" (2020). Graduate Theses, Dissertations, and Problem Reports. 7723.

https://researchrepository.wvu.edu/etd/7723

This Dissertation is protected by copyright and/or related rights. It has been brought to you by the The Research Repository @ WVU with permission from the rights-holder(s). You are free to use this Dissertation in any way that is permitted by the copyright and related rights legislation that applies to your use. For other uses you must obtain permission from the rights-holder(s) directly, unless additional rights are indicated by a Creative Commons license in the record and/ or on the work itself. This Dissertation has been accepted for inclusion in WVU Graduate Theses, Dissertations, and Problem Reports collection by an authorized administrator of The Research Repository @ WVU.

For more information, please contact researchrepository@mail.wvu.edu. 


\title{
Theory and Techniques of Convergence for Topological Transformation Group Actions
}

\author{
Murtadha Sarray \\ Dissertation Submitted \\ to the Eberly College of Arts and Sciences \\ at West Virginia University \\ in partial fulfillment of the requirements for the degree of \\ Doctor in Philosophy in \\ Mathematics
}

\author{
Edgar Fuller, Ph.D., Chair \\ Harvey Diamond, Ph.D. \\ Jerzy Wojciechowski, Ph.D. \\ Adam Halasz, Ph.D. \\ Ela Celikbas, Ph.D. \\ Department of Mathematics and Astronomy \\ Morgantown, West Virginia \\ 2020 \\ Keywords: \\ Proper map, Cartan and Proper G-Space, Slices \\ Copyright 2020 Murtadha Sarray
}




\section{Abstract \\ Theory and Techniques of Convergence for Topological Transformation \\ Groups}

\section{Murtadha Sarray}

In this dissertation, we present new set functions called strongly limit and strongly prolongation limit sets. We show the new sets, especially strongly prolongation limit sets, characterize proper action under an arbitrary setting. That is, we characterize proper action for wider class of proper $G$-spaces. Also, we show the new version of the sets could be derived from strongly exceptional sets which have been used as a good technique for the characterization of a proper maps. Moreover, we review properties of well-known limit sets and prolongations and properties for the new version of limit sets under an arbitrary setting on a $G$-space $X \mathrm{Next}$, we give characterizations for a proper action and a locally proper action using the new set functions and some relevant areas where one could use these sets to describe some topological properties for a space Xr algebraic properties for a group Gacting on XFollowing the technique of limit sets, we present a new version of action as weaker forms of the proper action called $w$-actions using convergence techniques. This approach yields new directions for the proof of some existing theorems which are then given as well as come counter-examples. We give some applications for our weaker forms and how convergence techniques could be fruitful to explain the relations among certain notions in the theory of $G$-spaces. Finally, according to our weaker forms, we characterize the notion of enough slice that has presented by H. Biller using $w$-Cartan action. 


\section{Contents}

0. Introduction ---

1. Fundamental Concepts---------_-

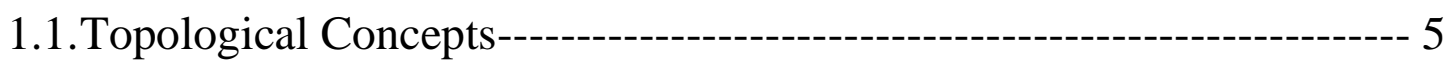

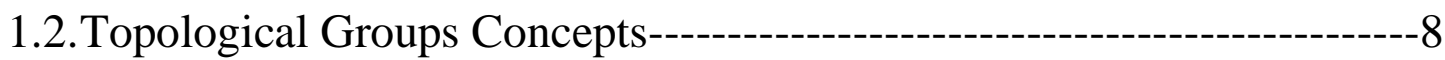

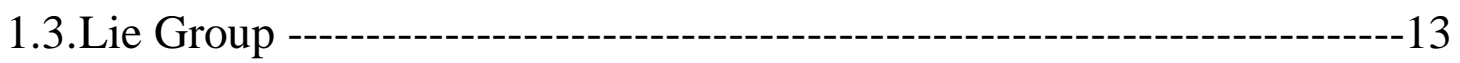

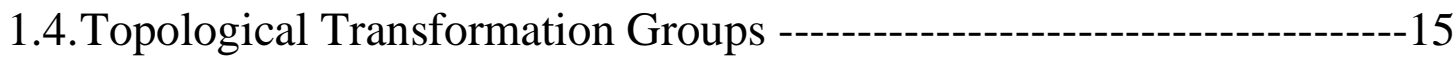

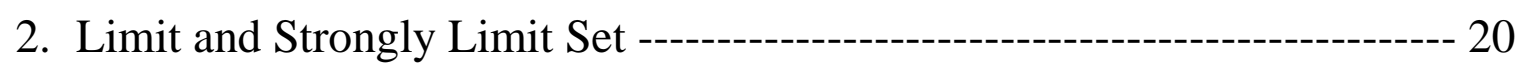

2.1.Basic Concepts and Convergence Techniques----------------------20

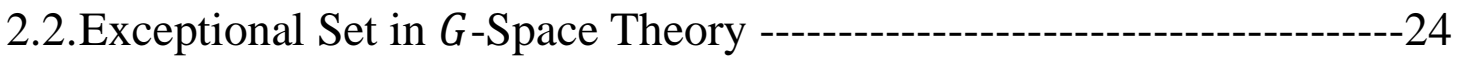

2.3.Strongly Exceptional Set in $G$-Space---------------------------------28

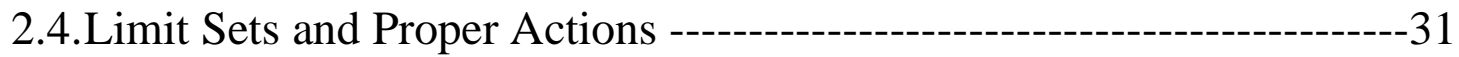

3. Weaker Forms of Proper Actions---

3.1 Three Kinds of Proper Actions------------------------------------------41

3.2 Biller's Cartan Action and Weaker Cartan Actions ----------------- 44

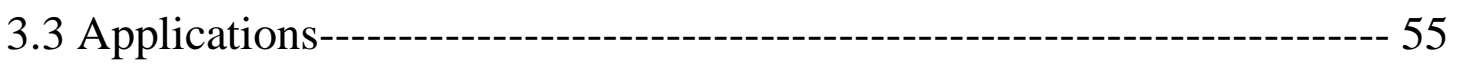

4. Convergence Techniques for Slices-------------------------------------- 59

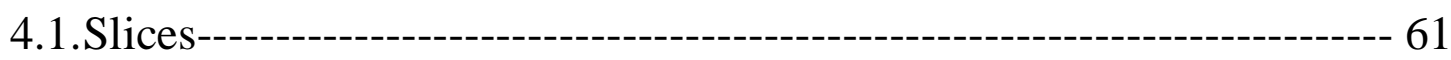

4.2.Slice in $w$-Actions------o- 64

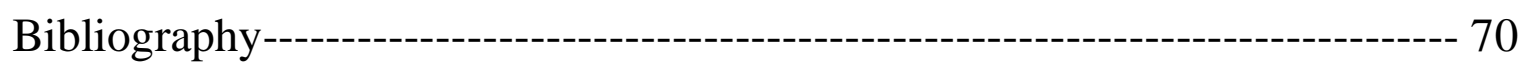




\section{Chapter 0}

\section{Introduction}

Limit and prolongation limit sets are used in the theory of dynamical systems to describe trajectory behaviors, especially their stability in their phase spaces [7]. It is well known within that theory ( $R$-spaces or $Z$-spaces), briefly, as a special case of a general theory called topological transformation groups ( $G$-space theory), so several studies have been conducted with these kinds of set functions to describe also properties of the general topological transformation groups.

In general, two versions of proper $G$-spaces were discovered in the middle of $19^{\text {th }}$ century. Palais in [28] introduces most of the basic concepts of $G$-spaces which under certain settings are taken with $G$ as a compact (Lie) group and $X$ a completely regular topological space. On the other hand, Bourbaki [11], introduces the concept of proper action for arbitrary topological groups and general topological spaces. Next Palais generalizes the class of a compact group action to wider one that is termed a proper action (or Palais proper) where $G$ is an arbitrary Hausdorff locally compact topological group and $X$ is a completely regular space. After Palais' work, many studies have arisen to investigate conditions that keep many notions no longer true in the sense of Palais such as the slice theorem, the conjugacy theorem, the equivariant embedding theorem and others. Some researchers have used these set functions as a technique to characterize a proper action groups on spaces under certain conditions. A fortiori one could use them also to investigate notions are related to proper action. 
In chapter one, we recall some basic background of topological concepts such as the separation axioms, compactness, local compactness, nets, connectedness, and paracompact spaces. In section two, we review many concepts of the theory of topological groups. For instance, we review nets in a topological group, locally compact groups and Lie groups which form the background of our work. In the last section, we concern ourselves with some notions of $G$-space theory. We recall the definitions and properties of proper mapping, Bourbaki proper action, and Palais proper action.

In chapter two, especially in section one, we recall some concepts of general topology theory such as, for example, quasi-compact mappings, proper mappings, and their characterizations by convergence techniques. In section two from the same chapter, we state the definitions of the limit set and its prolongation limit set under arbitrary settings. Also, we review properties and some counter examples. In section three, we present the definitions of the strongly limit set and its strongly prolongation limit set also under arbitrary settings. Moreover, we list properties of the new version of sets and give the main result in this chapter (Theorem 2.3.3) which is given as the following:

An action of a group $G$ on a space $X$ is proper if and only if the strongly prolongation set is the empty.

In the last section, we first recall some interesting results for proper and locally proper (Cartan) $G$-spaces in the sense of Palais setting and how limit sets could be good keys to describe the orbit space. Also, we present other results under weaker conditions such as the relations of close points and their stabilizers and close orbits and their kernels. Finally, we give a new proof for conjugacy neighborhood theorem. 
In chapter three, section one, we show how convergence techniques can describe well-known kinds of proper actions such as Bourbaki, Palais, and the stronger one that is presented by Buam, Connes and Higson. Moreover, we also show how the sets might be used to prove results concerning and a good technique to gain more knowledge about the local structure of $G$-spaces. In section two, we review the weaker conditions of the version of Cartan $G$-space that are introduced by Biller in [8] and show that these conditions could be replaced by a weaker condition (convergence condition) using set functions. Also, we give an example that shows that our condition is weaker than Biller's. Moreover, according to the weaker form of Cartan (we follow Biller), we introduce three kinds actions are called $w$-proper actions that are increasing strength. Furthermore, we investigate the relations and implications among the new version of the $w$-actions. Finally, in section three, an application for the weaker forms of $w$-actions on a topological group is given and we show some results from [5] that become immediately results of our forms and their proofs become short and understandable. By $G$-space $X$ it is meant an arbitrary topological group $G$ acts continuously on an arbitrary topological space $X$. We also follow Bourbaki for term "quasi-notion" is taken by a definition of the notion lacking Hausdorff axiom. Thus, by a quasi-compact, and a quasi-paracompact we mean these notions without $T_{2}$ property. Regular and completely regular spaces always possess the $T_{1}$ property.

Chapter four is divided into two sections, in the first one, we discuss the slice notion in $G$-space theory and give a motivation for the existence of slice theory. We rely there on the usual definition of the twisted product to describe an $H$-slice. In the second section, we characterize the enough slice notion, that has been presented by Biller [8], using the weaker form of $w$-Cartan action for $G$ as a locally compact group (not necessary Lie group) and $X$ as a completely regular 
space. As a result, we give the relation between Baum-Connes-Higson proper with our notion. 


\section{Chapter 1}

\section{Fundamental Concepts}

In this chapter we recall the basic background of topological spaces, topological groups, and topological transformation groups that form a base for all the notions that we shall present over next chapters.

\subsection{Topological Concepts}

1.1.1 Theorem. [25] Let $X$ be a topological space. Then the following are equivalent:

1. $X$ is $T_{1}$, that is, every two distinct points $x, y$ in $X$, each belong to an open set which does not contain the other.

2. Every singleton subset $\{P\}$ of $X$ is closed.

3. Every singleton subset $\{P\}$ of $X$ is the intersection of all open sets containing it.

1.1.2 Theorem. [11] Let $X$ be a topological space. Then the following are equivalent:

1. $X$ is $T_{2}$, that is, every pair of distinct points $x, y \in X$ belongs respectively to disjoint open sets. 
2. The intersection of the closed neighborhoods of any point of $X$ consists of that point alone.

3. The diagonal of the product space $X \times X$ is a closed set.

1.1.3 Definition. [14] A topological space $X$ is completely regular if whenever $F$ is a closed set in $X$ and $x \in X$ such that $x \notin F$, there is a continuous map $f: X \rightarrow$ $[0,1]$ such that $f(x)=0$ and $f(F)=1$.

1.1.4 Remark. In next chapter, when we mention a completely regular space $X$ we mean in addition that it agrees with the above definition and that it is also $T_{1}$, that is $T_{3 \frac{1}{2}}$.

1.1.5 Definition. [30] A topological space $X$ is locally compact if each point in $X$ has a compact neighborhood.

1.1.6 Definition. [30] A topological space $X$ is called regular provided for every closed subset $F$ of $X$, and a point $x \in X$ with $x \notin F$ there are two disjoint open neighborhoods $U$ and $V$ with $x \in U$ and $F \subseteq V$.

\subsubsection{Remark.}

1. In a $T_{2}$ locally compact space $X$ each point has a neighborhood whose closure is compact, then we often shall say that points of $X$ have a compact closure neighborhood. Over the next chapters, locally compact space is always meant $T_{2}$, otherwise we call it locally quasi-compact.

2. A $T_{2}$ locally compact space $X$ has a property that every point $x \in X$ there is a collection of neighborhoods $\left\{U_{n}\right\}$ of $x$ with $\bar{U}_{n+1} \subseteq U_{n}$ where $\bar{U}_{n+1}$ is compact for every positive integer $\mathrm{n}$. It is also important to refer here that the collection of neighborhoods above may not be neighborhood base for the point $x$.

1.1.8 Definition. [30] A set $D$ is a directed set if there is a relation $\leq$ on $D$ satisfying: 
1. $\alpha \leq \alpha$ for each $\alpha \in D$.

2. If $\alpha_{1} \leq \alpha_{2}$ and $\alpha_{2} \leq \alpha_{3}$ then $\alpha_{1} \leq \alpha_{3}$.

3. If $\alpha, \beta \in D$, then there is some $\gamma \in D$ with $\alpha \leq \gamma$ and $\beta \leq \gamma$.

1.1.9 Definition. [30] A net in a set $X$ is a map $x: D \rightarrow X$, where $D$ is a directed set. The image $x(\alpha)$ is a usually denoted by $x_{\alpha}$ and we often use the notation $\left\{x_{\alpha}\right\}_{\alpha \in D}$ or $\left\{x_{\alpha}\right\}$. A subnet of a net $x: D \rightarrow X$ is a composition $x \circ \varphi$, where $\varphi: M \rightarrow D$ is a map and $M$ is a directed set such that

1. $\varphi\left(\alpha_{1}\right) \leq \varphi\left(\alpha_{2}\right)$ whenever $a_{1} \leq \alpha_{2}$.

2. For each $\alpha \in D$, there is $\gamma \in M$ such that $\alpha \leq \varphi(\gamma)$. For $\gamma \in M$ the image $x \circ \varphi(\gamma)$ is written as $x_{\alpha_{\gamma}}$.

1.1.10 Definition. [30] Let $\left\{x_{\alpha}\right\}$ be a net in a topological space $X$. Then $\left\{x_{\alpha}\right\}$ converges to $x \in X$ (written $x_{\alpha} \rightarrow x$ ) provided it is eventually in every neighborhood of $x$. That is, for every neighborhood $U$ of $x$ there is an $\alpha_{\circ} \in D$ such that $x_{\alpha} \in U$ for each $\alpha \geq \alpha_{\circ}$. We say $\left\{x_{\alpha}\right\}$ has a point $x$ as a cluster point provided $\left\{x_{\alpha}\right\}$ is frequently in every neighborhood of $x$, i.e., for every neighborhood $U$ of $x$ and every $\alpha_{\circ} \in D$, there is some $\alpha \in D$ with $\alpha \geq \alpha_{\circ}$ such that $x_{\alpha} \in U$.

1.1.11 Theorem. [30] A net $\left\{x_{\alpha}\right\}$ has $y$ as a cluster point if and only if has a subnet which converges to $\mathrm{y}$.

1.1.12 Theorem. [30] If $E \subset X$, then $x \in \bar{E}$ if and only if there is a net $\left\{x_{\alpha}\right\}$ in $E$ with $x_{\alpha} \rightarrow x$.

1.1.13 Theorem. [30] Let $f: X \rightarrow Y$ be a map. Then $f$ is continuous at $x_{\circ}$ in $X$ if and only if whenever $x_{\alpha} \rightarrow x_{\circ}$ in $X$, then $f\left(x_{\alpha}\right) \rightarrow f\left(x_{\circ}\right)$ in $Y$.

1.1.14 Theorem. [30] For a topological space $X$, the following are equivalent:

a) $X$ is called compact ( $\cong$ for every open cover of $X$ has a finite subcover).

b) Every net in $X$ has a cluster point. 


\subsubsection{Remark.}

1. A net $\left\{x_{\alpha}\right\}$ in $X$ is said to have no a convergent subnet in $X$ is written as $x_{\alpha} \rightarrow \infty$ if and only if every subnet of $\left\{x_{\alpha}\right\}$ has no a limit point [30].

2. If $X$ is $T_{2}$, then every convergent net in $X$ has a unique limit [30].

3. Over the next chapters, compact space will be always $T_{2}$. Otherwise, we call it quasi-compact.

4. From now on, a map will always mean a continuous mapping.

1.1.16 Definition. [24] Let $X$ be a topological space. A collection $\mathcal{A}$ of subsets of $X$ is said to be locally finite in $X$ if every point of $X$ has a neighborhood that intersects only finitely many elements of $\mathcal{A}$.

1.1.17 Lemma. [24] Let $\mathcal{A}$ be a locally finite collection of subsets of $X$. Then:

a. Any subcollection of $\mathcal{A}$ is locally finite.

b. The collection of the closures of the elements of $\mathcal{A}$ is locally finite.

c. $\overline{\mathrm{U}_{A \in \mathcal{A}} A}=\mathrm{U}_{A \in \mathcal{A}} \bar{A}$.

1.1.18 Definition. [24] Let $\mathcal{A}$ be a collection of subsets of the space $X$. A collection $\mathcal{B}$ of subsets of $X$ is said to be a refinement of $\mathcal{A}$ (or is said to refine $\mathcal{A}$ ) if for each element $B$ of $\mathcal{B}$ there is an element $A$ of $\mathcal{A}$ containing $B$. If the elements of $\mathcal{B}$ are open sets, we call $\mathcal{B}$ an open refinement of $\mathcal{A}$; if they are closed sets, we call a closed refinement.

1.1.19 Definition. [24] A space $X$ is paracompact if every open covering $\mathcal{A}$ of $X$ has a locally finite open refinement $\mathcal{B}$ that covers $X$.

1.1.20 Example. The set of real numbers $\mathbb{R}$ with the usual topology is paracompact. If $\mathcal{A}$ is an open cover of $\mathbb{R}$. Consider a collection of open intervals $O_{n}=(-n, n), n=0,1,2, \ldots$ where $O_{0}=\emptyset$. Since $\bar{O}_{n}$ is compact, then there is a 
finite number of members of $\mathcal{A}$, say $A_{1}, A_{2}, \ldots, A_{r_{n}}$, covers $\bar{O}_{n}$. Put $B_{i}=A_{i} \cap$ $\left(\mathbb{R} \backslash \bar{O}_{n-2}\right)$, and $\mathcal{B}_{n}=\left\{B_{1}, B_{2}, \ldots, B_{n}\right\}$. Then the collection $\mathcal{B}=\cup \mathcal{B}_{n}$ is locally finite and a refinement of $\mathcal{A}$.

\subsection{Topological Group Concepts}

1.2.1 Definition. [18] A topological group is a set $G$ with two structures:

1. $G$ is a group.

2. $G$ is a topological space.

such that the two structures are compatible; that is, the multiplication law $\mu: G \times G \rightarrow G$ and the inversion law $v: G \rightarrow G^{-1}$ are both continuous.

\subsubsection{Remark.}

1. The two conditions in definition (1.2.1) are equivalent to the fact that the map $\tau: G \times G \rightarrow G$ is defined by $\tau\left(g_{1}, g_{2}\right)=g_{1} g_{2}^{-1}$ is continuous.

2. A continuous map $l_{g}: G \times G \rightarrow G$ defined by $x \rightarrow g x$ is called the left translation by $g$. This map has inverse $l_{g^{-1}}$ which is also continuous.

Moreover, $l_{g}$ is a homeomorphism. Similarly, all right translations $r_{g}: G \rightarrow$ $G$ are homeomorphisms.

3. A topological space $X$ which satisfies the property that for every pair of points $x, y \in X$, then there is a homeomorphism on $X$ sending $x$ to $y$ is called homogeneous. Therefore, every topological group is homogeneous. A map $l_{y x^{-1}}\left(\right.$ or $\left.r_{x^{-1} y}\right)$ send a point $x$ into $y$.

4. Every group admits a topological group structure with discreate topology.

1.2.3 Proposition. [18] Let $G$ be a topological group, and $A, B$ be subsets of $G$.

Then for $g \in G$ we have: 
1. $A g=A r_{g}=\{a g: a \in A\} ; A g$ is called the right translate of $A$ by $g$.

2. $g A=A l_{g}=\{g a: a \in A\} ; g A$ is called the left translate of $A$ by $g$.

3. $A B=\bigcup_{b \in B} A b=\bigcup_{a \in A} a B$.

4. $A^{-1}=\left\{a^{-1}: a \in A\right\}$.

1.2.4 Proposition. [18] Let $G$ be a topological group and $A, B \subset G$ and $g \in G$. Then:

1. If $A$ is open, then $A g$ and $g A$ are open.

2. If $A$ is closed, the $A g$ and $g A$ are closed.

3. If $A$ is open, then $A B$ and $B A$ are open.

4. If $A$ is closed and $B$ is finite, then $A B$ and $B A$ are closed.

1.2.5 Remark. Let $G$ be a topological group and $\mathcal{F}$ be a fundamental system of neighborhoods of the identity element $e$ in $G$. Then for every $g \in G$ the collection $\mathcal{F} g=\left\{U g: U_{\in} \mathcal{F}\right\}$ is a fundamental system of open neighborhoods of $g$.

1.2.6 Proposition. [18] Let $\mathcal{F}$ be a fundamental system of open neighborhoods of the identity element $e$. Then

1. If $U, V \in \mathcal{F}$, there is $W \in \mathcal{F}$ such that $W \subseteq U \cap V$.

2. If $g \in U \in \mathcal{F}$, then there is $V \in \mathcal{F}$ such that $g V \subseteq U$.

3. If $U \in \mathcal{F}, g \in G$, then there is $V \in \mathcal{F}$ such that $g^{-1} V g \subseteq U$.

4. If $U \in \mathcal{F}$, then there is $V \in \mathcal{F}$ such that $V V^{-1} \subseteq U$.

1.2.7 Remark. Any fundamental system of open neighborhoods of the identity element e, say $\mathcal{F}$, also satisfies: $\forall U \in \mathcal{F}, \exists V, W \in \mathcal{F}$ such that $V^{-1} \subseteq U$ and $W^{2} \subseteq$ $U$.

1.2.8 Proposition. Let $G$ be a topological group. Then $G$ is regular.

Proof. Take a closed subset $F$ of $G$ and a point $e \in G$ such that $e \notin F$. Then there is a basic neighborhood $U \in \mathcal{F}$ such that $U U^{-1} \subseteq F^{c}$ or $U U^{-1} \cap F=\emptyset$. Then $U \cap$ 
$F U=\emptyset . F U$ is a neighborhood of $F$. By homogenous property of $G$. Therefore, $G$ is regular.

1.2.9 Proposition. A topological group $G$ is $T_{1}$ if and only if the set $\{e\}$ is closed.

Proof. If $\{e\}$ is closed then for every point $g \in G$, the set $g\{e\}=\{g\}$ is closed.

Thus, $G$ is $T_{1}$. The other side of proof is obvious.

1.2.10 Proposition. Let $G$ be a topological group and $H$ be a subgroup of $G$. Then the following properties hold:

1. $H$ is also a topological subgroup. The topology on $H$ is just the relative topology of the topology of $G$.

2. The left (or right) coset set $G / H=\{g H: g \in G\}$ is endowed, in a natural way, by a quotient topology induced by the canonical quotient map $q: G \rightarrow$ $G / H$. This topology makes the map $q$ is open and continuous and $G / H$ is regular.

3. If $H$ is a normal subgroup of $G$, then the coset space is also a topological group.

4. The coset space $G / H$ is $T_{2}$ if and only if $H$ is a closed subgroup of $G$.

5. If $H$ is an open subgroup, then it is also closed.

Proof. It is obvious.

1.2.11 Proposition. Let $G$ be a topological group and $\left\{g_{\alpha}\right\}$ be a net in $G$. Then the following hold.

1. $\left\{g g_{\alpha}\right\}$ (or $\left\{g_{\alpha} g\right\}$ ) is a net in $G$ for each $g \in G$.

2. If $g_{\alpha} \rightarrow e$, then $g g_{\alpha} \rightarrow g$ (or $g_{\alpha} g \rightarrow g$ ) for each $g \in G$.

3. If $g_{\alpha} \rightarrow \infty$, then $g g_{\alpha} \rightarrow \infty$ (or $g_{\alpha} g \rightarrow \infty$ ) for each $g \in G$.

4. If $g_{\alpha} \rightarrow \infty$, then $g^{-1} \rightarrow \infty$.

Proof. The proof of all these statements follows from the continuity of multiplication and inversion maps. 
1.2.12 Definition. A topological group $G$ is called quasi-compact provided the underlying topological space of $G$ is a quasi-compact, that is, for every open cover of $G$ has a finite subcover. Moreover, $G$ is called locally quasi-compact whenever $G$ is a locally quasi-compact as topological space and this is equivalent to that there is a quasi-compact neighborhood of the identity element $e$ of $G$.

1.2.13 Definition. A topological group $G$ is a called connected provided the underlying topological space $G$ is connected, that is, there are not two disjoint clopen subsets $U$ and $V$ of $G$ separating $G$. Moreover, $G$ is called locally connected if each point $g$ in $G$ has a connected neighborhood. If the only connected subspaces of $G$ are the singletons, then $G$ is called totally disconnected.

\subsubsection{Components and identity components}

Let $G$ be a topological group and let $R$ be a relation on $G$ is defined by for every $x, y \in G: x R y$ if and only if there is a connected subspace of $G$ containing both $x$, and $y . R$ is an equivalence relation on $G$. Therefore, $R$ decomposes $G$ into maximal disjoint connected subspaces are called components. The component that contains the identity element $e$ is called the identity component is denoted by $G_{\circ}$. In general, the components are closed but not open.

\subsubsection{Remark.}

1. The identity component $G_{\circ}$ is a closed normal subgroup of $G$.

2. The quotient $G / G_{\circ}$ is always a totally disconnected topological group. 


\subsubsection{Some properties of quasi-compact and locally quasi-compact group.}

If $G$ is a topological group and $H$ is a subgroup of $G$, then the following hold:

1. If $G$ is locally quasi-compact, then every quasi-compact subset $K$ of $G$ has a quasi-compact neighborhood.

2. If $G$ is quasi-compact (or locally quasi-compact) group, and $H$ is closed, then $H$ and $G / H$ are quasi-compact (locally quasi-compact).

3. If $G$ is a quasi-compact group and $H$ is closed. Then for $g \in G, g H g^{-1} \subseteq H$ if and only if $g \mathrm{Hg}^{-1}=H .[\mathbf{1 2}]$

4. The closure $\bar{H}$ is also a subgroup of $G$. Moreover, if $H$ is normal then so is $\bar{H}$.

5. If $C$ is a quasi-compact subset of $G$, then $\bar{C}$ is quasi-compact since $\bar{C}$ is the image of $C$ quotient map $G \rightarrow G / \overline{\{e\}}$. [17]

\subsection{Lie Groups}

In this section, we recall the concept of Lie group and some its relative properties, and we will not go deeply in this concept. However, it forms background for what we study in the chapter four.

1.3.1 Definition. [22] Let $M$ be a topological space and $\mathbb{R}^{n}$ be $n$-dimensional Euclidean space. A pair $(U, \varphi)$ consisting of an open set $U$ in $M$ and a homeomorphism $\varphi$ of $U$ in $M$ onto an open set in $\mathbb{R}^{n}$, is called a chart (or coordinate neighborhood) of $M$. When we write

$$
\varphi(x)=\left(\varphi_{1}(x), \varphi_{2}(x), \ldots, \varphi_{n}(x)\right), \quad x \in U,
$$


$\varphi_{i}(x)$ is called $i t h$ coordinate function. If a point $x$ of $M$ lies in $U$, then we say that $(U, \varphi)$ is a chart at $x$.

A collection of charts $\left\{\left(U_{\alpha}, \varphi_{\alpha}\right)\right\}$ is called an atlas (or system of coordinate neighborhoods) on $M$ if $\left\{U_{\alpha}\right\}$ forms a cover of $M$. The atlas is smooth if each pair of indices $\alpha, \beta$ with $U_{\alpha} \cap U_{\beta} \neq \varnothing$, the map

$$
\varphi_{\beta} \cdot \varphi_{\alpha}^{-1}: \varphi_{\alpha}\left(U_{\alpha} \cap U_{\beta}\right) \rightarrow \varphi_{\alpha}\left(U_{\alpha} \cap U_{\beta}\right)
$$

is smooth (that is, of the class $\mathbb{C}^{\infty}$ ).

1.3.2 Definition. [22] Two smooth atlases $\left\{\left(U_{\alpha}, \varphi_{\alpha}\right)\right\}$ and $\left\{\left(V_{\alpha}, \varphi_{\alpha}\right)\right\}$ are said to be equivalent if the union $\left\{\left(U_{\alpha}, \varphi_{\alpha}\right)\right\} \cup\left\{\left(V_{\alpha}, \varphi_{\alpha}\right)\right\}$ is smooth, i.e., if the map

$$
\varphi_{\beta} \cdot \varphi_{\alpha}^{-1}: \varphi_{\alpha}\left(U_{\alpha} \cap V_{\beta}\right) \rightarrow \varphi_{\alpha}\left(U_{\alpha} \cap V_{\beta}\right)
$$

is a diffeomorphism for each pair $(\alpha, \beta)$ with $U_{\alpha} \cap V_{\beta} \neq \emptyset$. An equivalence class of atlases on $M$ is called a differential or smooth structure on $M$ and we denote the equivalence class of an atlas $\left\{\left(U_{\alpha}, \varphi_{\alpha}\right)\right\}$ by $\left[\left(U_{\alpha}, \varphi_{\alpha}\right)\right]$.

1.3.3 Definition. [20] A smooth manifold is a pair $\left(M,\left[\left(U_{\alpha}, \varphi_{\alpha}\right)\right]\right)$ where $M$ is a Hausdorff space satisfying the second axiom of countability and $\left[\left(U_{\alpha}, \varphi_{\alpha}\right)\right]$ is a smooth structure on $M$. We also say that $\left(M,\left[\left(U_{\alpha}, \varphi_{\alpha}\right)\right]\right)$ is a smooth $n$-manifold when we want to emphasize the dimension of $\mathbb{R}^{n}$.

1.3.4 Definition. [22] $G$ is a Lie group provided that:

1) $G$ is a Manifold.

2) $G$ is a group.

3) The group operation is smooth, that is, the maps $\alpha: G \times G \rightarrow G$ and $\beta: G \rightarrow$ $G$ defined by $\alpha(g, h)=g h$ and $\beta(g)=g^{-1}$ both smooth. Here $G \times G$ is endowed with the structure of the product manifold.

1.3.5 Example. $\operatorname{Gl}(n, \mathbb{C})$ is one of the classic Lie groups. 


\section{Properties of Lie groups}

1.3.6 Theorem. [20] A closed subgroup $H$ of a Lie group $G$ is an embedded Lie subgroup.

1.3.7 Theorem. [20] If $H$ is a closed subgroup of a Lie group $G$, then there exists a unique smooth structure on the space $G / H$ such that:

1) The projection $q: G \rightarrow G / H$ is smooth.

2) The projection $q: G \rightarrow G / H$ has a smooth local cross-section.

1.3.8 Corollary. [20] If $H$ is a closed normal subgroup of a Lie group $G$, then $G / H$ is a Lie group and the natural projection $q: G \rightarrow G / H$ is a Lie group homomorphism.

1.3.9 Proposition. [20] Every Lie group $G$ is locally compact.

1.3.10 Proposition. [20] Every Lie group $G$ is locally connected.

1.3.11 Definition. [19] A topological group has no small subgroups (NSS) if there exists a neighborhood $U$ of the identity element $e$ such that the only subgroup in $U$ is $\{e\}$.

1.3.12 Proposition. [19] Every Lie group $G$ is NSS.

1.3.13 Proposition. [19] Locally compact $G$ of $N S S$ property is a Lie group.

\subsection{Topological Transformation Groups}

1.4.1 Definition. [12] A topological transformation group is a triple $(G, X, \varphi)$ where $G$ is a topological group and $X$ is a topological space and $\varphi: G \times X \rightarrow X$ is a continuous map such that:

a. $\varphi\left(g_{1}, \varphi\left(g_{2}, x\right)\right)=\varphi\left(g_{1} g_{2}, x\right)$ for every $g_{1}, g_{2} \in G, x \in X$.

b. $\varphi(e, x)=x$ for every $x \in X$, where $e$ the identity element of $G$.

\subsubsection{Remark.}


1. The map $\varphi$ is called an action of $G$ on $X$ and the space $X$ together with $\varphi$ is called a $G$-space (or more precisely left $G$ - space).

2. Since $\varphi$ is understood from the context we may use a notation $g x$ for $\varphi(g, x)$ and $g_{1}\left(g_{2} x\right)=\left(g_{1} g_{2}\right) x$ for $\varphi\left(g_{1}, \varphi\left(g_{2}, x\right)\right)=\varphi\left(g_{1} g_{2}, x\right)$.

3. For $H \subseteq G$ and $A \subseteq X$ we write $H A=\left\{g a \mid g \in H, a_{\in} A\right.$ for $\varphi(H, A)$.

4. A subset $A \subseteq X$ is said to be invariant under $G$ if $G A=A$.

5. For $g_{\in} G$, a map $\varphi_{g}: X \rightarrow X$ is defined by $\varphi_{g}(x)=\varphi(g, x)=g x$ is a continuous. Also, $\varphi_{g_{1}} \varphi_{g_{2}}=\varphi_{g_{1} g_{2}}$ and $\varphi_{e}=I_{X}$ is the identity map of $X$. A map $\varphi_{g}$ has an inverse continuous map $\varphi_{g^{-1}}$. Therefore, for every $g \in G$, the map $\varphi_{g}$ is a homeomorphism of $X$.

6. The set $\Gamma(X)=\left\{\varphi_{g}: g \in G\right\}$ forms a group under maps composition, and there is a natural group homomorphism $\Psi: G \rightarrow \Gamma(X)$ is given by $g \rightarrow \varphi_{g}$. Therefore, an action of a topological group $G$ on a space $X$ is determined by a fixed homomorphism $\Theta$ from $G$ into the group of all homeomorphisms of $X \operatorname{Homeo}(X)$ is given by $g x=f(x)$, where $f \in \operatorname{Homeo}(X)$. The kernel of the action is exactly the kernel of the map $\Theta$.

\subsubsection{Definitions and notations.}

For a $G$-space $X$, and $x \in X$ a subset $G x=\{g x: g \in G\}$ is called the $G$-orbit through a point $x$. Let $R$ be a relation on $X$ is defined such that $(x, y) \in R$ if and only if there is $g \in G$ such that $g x=y$, that is, $(x, y) \in R$ if and only if both $x$, and $y$ belong to the same orbit. Thus, $G$-orbits form a partition on $X$. The set of all $G$-orbits is denoted by $X / G$ and it is called orbit space. One may endow the orbit space with a quotient topology and a natural open quotient map $\pi: X \rightarrow X / G$ which sends every point $x$ into its orbit $G x$. For a point $x \in X$, a subset $G_{x}=\{g \in$ $G: g x=x\}$ is a subgroup of $G$ is called the stabilizer subgroup over $x$. A kernel of 
an action $\Theta$ on a space $X$ can be defined as $\operatorname{ker} \Theta=\bigcap_{x \in X} G_{x}$. An action of $G$ on a space $X$ is called free if $G_{x}=\{e\}$ for every $x \in X$. It is called effective if $\operatorname{ker} \Theta=$ $\{\mathrm{e}\}$ while it called transitive if the orbit space $X / G$ is just one element.

1.4.4 Definition. [12] An equivariant map (continuous) (or a $G$-map) of left (right) $G$-spaces $X$ and $Y$ is a map $f: X \rightarrow Y$ that is preserved the action operation of $G$, that is, for every $g \in G$, and $x \in X$, then $f(g x)=g f(x)$. An equivariant map which is also homeomorphism is called an equivalence.

\subsubsection{Some properties of equivariant maps}

1. If $Y$ is a $G$-space and $X$ is a topological space such that there is a map $f: X \rightarrow$ $Y$ with the property $f(g x)=g f(x)$, for every $g \in G$, and $x \in X$, then the map $f$ admits an action on $X$ by $G$ is given by $g x=g f(x)$.

2. If $f: X \rightarrow Y$ is an equivariant map and $x \in X$, then $f(G x) \subseteq G f(x)$, that is, $f$ maps an orbit in $X$ into an orbit in $Y$. Moreover, $f\left(G_{x}\right) \subseteq G_{f(x)}$.

3. A $G$-map $f$ is called isovariant if it is one to one on each orbit, that is, $f\left(G_{x}\right)=G_{f(x) \cdot[28]}$

\section{Proper mapping and proper action}

1.4.6 Definition. [30] A map $f: X \rightarrow Y$ of topological space $X$ and $Y$ is called closed (open) if the directed image of $f$ for every closed subset of $X$ is closed (open) in $Y$.

1.4.7 Definition. [11] Let $f$ be a mapping of a topological space $X$ into a topological space $Y$. $f$ is said to be proper if $f$ is a continuous and the mapping $f \times i_{Z}: X \times Z \rightarrow Y \times Z$ is closed, for every topological space $Z$. 
1.4.8 Proposition: [11] Let $f: X \rightarrow Y$ be a continuous injection. Then the following three statements are equivalent:

1. $f$ is proper.

2. $f$ is closed.

3. $f$ is a homeomorphism of $X$ onto a closed subset of $Y$.

1.4.9 Theorem. [11] Let $f: X \rightarrow Y$ be a mapping. Then the following four statements are equivalent:

1. $f$ is proper.

2. $f$ is closed and $f^{-1}(y)$ is quasi-compact for every $y \in Y$.

3. If $\mathcal{F}$ is a filter on $X$ and if $y \in Y$ is a limit point of $f(\mathcal{F})$, then there is a cluster point $x$ of $\mathcal{F}$ such that $f(x)=y$.

4. If $\mathcal{U}$ is an ultrafilter on $X$ and if $y \in Y$ is a limit point of the ultrafilter base $f(\mathcal{U})$, then there is a limit point $x$ of $\mathcal{U}$ such that $f(x)=y$.

1.4.10 Definition. [29] Let $X$ be a $G$ - space. A subset $A$ of $X$ is said to be thin relative to a subset $B$ of $X$ if the closure of the set $((A, B))=\left\{g_{\in} G \mid g A \cap B \neq \varphi\right\}$ is quasi-compact in $G$, and we will say that $A$ and $B$ are relatively thin. If $A$ is thin relative to itself then it is called thin. $X$ is called Cartan if every point $x \in X$ has a thin neighborhood.

1.4.11 Remark. If $X$ is a $G$-space and $A, B$ are subsets of $X$. Then

1. Since $(g A \cap B)=g\left(A \cap g^{-1} B\right)$ it follows that if $A$ is thin relative to $B$, then $B$ is thin relative to $A$.

2. Since $\left(g g_{1} A \cap g_{2} B\right)=g_{2}\left(g_{2}^{-1} g g_{1} A \cap B\right)$ it follows that if $A$ and $B$ are relatively thin, then so are any their translates $g_{1} A$ and $g_{2} B$.

3. If $A$ and $B$ are relatively thin and $K \subset A, M \subset B$, then $K$ and $M$ are relatively thin.

4. If $K_{1}, K_{2}$ are quasi-compact subsets of $X$. Then $\left(\left(K_{1}, K_{2}\right)\right)$ is closed in $G$. 
5. If $K_{1}$ and $K_{2}$ are quasi-compact subsets of $X$ such that $K_{1}$ and $K_{2}$ are relatively thin, then $\left(\left(K_{1}, K_{2}\right)\right)$ is quasi-compact.

6. If $U$ is a neighborhood of $x$ in $X$, then $((U, U))$ is a neighborhood of the identity element $e$ in $G$. So, if $X$ is Cartan $G$-space, then it essential that $G$ is locally quasi-compact.

7. If $A$, and $B$ are subsets of $X$, then $((A, B))^{-1}=((B, A))$.

8. If $A$, and $B$ are subsets of $X$ and $g \in G$, then $((A, g B))=g((A, B))$ and $((A, g B))=((A, B)) g^{-1}$.

1.4.12 Definition. (Bourbaki Proper) Let $G$ be a topological group operating (acting) continuously on a topological space $X . G$ is said to operate (act) properly on $X$ if the mapping $\theta:(s, x) \rightarrow(x, s x)$ of $G \times X$ into $X \times X$ is proper.

1.4.13 Proposition. [11] If a topological group $G$ operates properly on a topological space $X$, then the orbit space $X / G$ is $T_{2}$.

1.4.14 Definition. (Palais Proper) A subset $S$ of a $G$-space $X$ is a small subset of $X$ if each point of $X$ has a neighborhood which is thin relative to $S$. If $G$ is a locally compact ( $T_{2}$ is included) and $X$ is a completely regular, then $G$ acts properly on $X$ provided that every point $x \in X$ has small neighborhood.

1.4.15 Remark. If $X$ is a $G$-space, in the sense of Palais, then the following hold:

1. For each $x \in X$, then $G x$, orbit of $x$, is closed in $X$.

2. The stabilizer subgroup $G_{x}$ is compact for every $x \in X$.

3. Palais proper is Bourbaki proper and Cartan. 


\section{Chapter Two}

\section{Limit and Strongly Limit Sets}

\subsection{Basic Concepts and Convergence Techniques.}

2.1.1 Definition. [11] A map $f: X \rightarrow Y$ of topological spaces $X$ and $Y$ is called quasi-compact if a preimage of every quasi-compact subset of $Y$ is a quasi-compact subset of $X$.

2.1.2 Definition. Let $x_{\alpha}$ be a net in a topological space $X$. A subset $\operatorname{clu}\left(x_{\alpha}\right)$ of $X$ is the set of all limit points of subnets of the net $x_{\alpha}$. If the net $x_{\alpha}$ has no convergent subnet, that is, $x_{\alpha} \rightarrow \infty$, then is clear that $\operatorname{clu}\left(x_{\alpha}\right)=\emptyset$.

2.1.3 Proposition. If $x_{\alpha}$ is a net in space $X$, then the set $\operatorname{clu}\left(x_{\alpha}\right)$ is a closed subset of $X$.

Proof. Take $z \in \overline{\operatorname{clu}\left(x_{\alpha}\right)}$, there is a net $z_{\alpha} \in \operatorname{clu}\left(x_{\alpha}\right)$ and $z_{\alpha} \rightarrow z$. For each $\alpha$, there is a net $z_{\gamma}^{\alpha} \rightarrow z_{\alpha}$. Then there is a diagonal subnet $z_{\gamma}^{\gamma}$ of the net $z_{\alpha}$ which converges to $z$. therefore, $z \in \operatorname{clu}\left(x_{\alpha}\right)$ and $\operatorname{clu}\left(x_{\alpha}\right)$ is closed.

2.1.4 Theorem. Let $f: X \rightarrow Y$ be a continuous mapping. Then the following are equivalent:

(i) $f$ is proper.

(ii) If $\left\{x_{\alpha}\right\}$ is a net in $X$ and $y$ is a cluster point of a net $f\left(x_{\alpha}\right)$, then there is a point $x$ in $X$ such that $x$ is a cluster point of $\left\{x_{\alpha}\right\}$ with $f(x)=y$.

Proof. If $f$ is proper. Take $\left\{x_{\alpha}\right\}_{\alpha \in D}$ a net in $X$ and $y$ is a point in $Y$ such that $f\left(x_{\alpha}\right) \propto y$, where the notation $\propto$ meant that the net $f\left(x_{\alpha}\right)$ has $y$ as cluster point. 
Put for every $\gamma \in D$ a subset $E_{\gamma}=\left\{x_{\alpha} \mid \alpha \geq \gamma\right\}$. It is easy to check that the family $\left\{E_{\alpha}\right\}_{\alpha \in D}$ satisfies the property of finite intersection. Since $f$ is closed, we have $f\left(\bar{E}_{\alpha}\right)=\overline{f\left(E_{\alpha}\right)}$ for all $\alpha \in D$. To show that for all $\alpha \in D, y \in \overline{f\left(E_{\alpha}\right)}$. Let $U$ be a neighborhood of $y$ and $\alpha \in D$. Then there is $\gamma \in D$ such that $\gamma \geq \alpha$ and $f\left(x_{\gamma}\right) \in$ $U$. Since $f\left(x_{\gamma}\right) \in f\left(E_{\alpha}\right)$, then $f\left(E_{\alpha}\right) \cap U \neq \emptyset$ and hence $y \in \overline{f\left(E_{\alpha}\right)}$ for all $\alpha \in$ $D$. For every $\alpha \in D, \bar{E}_{\alpha} \cap f^{-1}(y)$ is non-empty closed subset of $f^{-1}(y)$ which is a quasi-compact. The family $\left\{\bar{E}_{\alpha} \cap f^{-1}(y)\right\}_{\alpha \in D}$ satisfies the finite intersection property, that is, $\cap\left\{\bar{E}_{\alpha} \cap f^{-1}(y)\right\}=\cap \bar{E}_{\alpha} \cap f^{-1}(y) \neq \varnothing$. Then there is a point $x \in X$ such that $x \in \cap \bar{E}_{\alpha}$ and $x \in f^{-1}(y)$. We claim that $x_{\alpha} \propto x$. Take $U$ to be a neighborhood of $x$ in $X$ and $\delta \in D$. Then there is $\alpha \in D$ and $\alpha \geq \delta$ such that $E_{\alpha} \cap$ $U \neq \varnothing$. Thus, there is $\tau \in D$ with $\tau \geq \alpha$ and $x_{\tau} \in E_{\alpha} \cap U$. Therefore $x_{\alpha} \propto x$ and $f(x)=y$. Conversely, if the condition (ii) is satisfied, then $f^{-1}(y)$ is quasicompact for all $y \in Y$, otherwise there is a net $\left\{x_{\alpha}\right\}$ in $f^{-1}(y)$ with $x_{\alpha} \rightarrow \infty$ and $f\left(x_{\alpha}\right) \rightarrow y$ which is a contradiction. To show that $f$ is closed. Take a closed subset $F$ of $X$ and $y \in \overline{f(F)}$, then there is a net $\left\{x_{\alpha}\right\}$ in $F$ such that $f\left(x_{\alpha}\right) \rightarrow y$. By hypotheses $\left\{x_{\alpha}\right\}$ has a cluster point, say $x \in X$ such that $f(x)=y$. Since $F$ is closed then $x \in F$. Therefore, $f(x)=y \in f(F)$ and $f$ is proper.

Al Sarray M.J. in [1] has presented a characterization for a proper mapping called an exceptional set for a map under the Hausdorff assumption on a codomain space $Y$.

2.1.5 Definition. [1] (Exceptional Set): Let $f: X \rightarrow Y$ be a map. We define the set:

$$
E_{f}=\left\{y_{\in} Y: \exists \text { a net }\left\{x_{\alpha}\right\} \text { in } X \text { with } x_{\alpha} \rightarrow \infty \text { and } f\left(x_{\alpha}\right) \rightarrow y\right\}
$$


We call $E_{f}$ the exceptional set of $f$.

2.1.6 Definition. [2] (Strongly Exceptional Set): Let $f: X \rightarrow Y$ be a map. We define the set:

$$
S E_{f}=\left\{y_{\in} Y: \exists \text { a net }\left\{x_{\alpha}\right\} \text { in } X \text { with } f\left(x_{\alpha}\right) \rightarrow y \text { and } \operatorname{cls}\left(x_{\alpha}\right) \cap f^{-1}(y)=\emptyset\right\}
$$

We call $S E_{f}$ the strongly exceptional set of $f$.

2.1.7 Remark. In general, $E_{f} \subseteq S E_{f}$, with equality demonstrated in the following proposition.

2.1.8 Proposition. Let $f: X \rightarrow Y$ be a continuous map and $Y$ is $T_{2}$. Then $E_{f}=S E_{f}$.

Proof. It suffices to show that $S E_{f} \subseteq E_{f}$. Take $z \in S E_{f}$, then there is a net $x_{\alpha}$ in $X$ such that $f\left(x_{\alpha}\right) \rightarrow z$ and $\operatorname{clu}\left(x_{\alpha}\right) \cap f^{-1}(z)=\emptyset$. Suppose that there is $x \in$ $\operatorname{clu}\left(x_{\alpha}\right)$. Then there is a subnet $x_{\alpha_{\beta}}$ of $x_{\alpha}$ such that $x_{\alpha_{\beta}} \rightarrow x$. By continuity of $f$, we have $f\left(x_{\alpha_{\beta}}\right) \rightarrow f(x)$ and by $T_{2}$ property, we have $f(x)=z$. Thus, we have $x \in f^{-1}(z)$ which is a contradiction. Therefore, $\operatorname{clu}\left(x_{\alpha}\right)=\emptyset$ and $x_{\alpha} \rightarrow \infty$.

2.1.9 Proposition. Let $f: X \rightarrow Y$ be a map of topological spaces. Then the set $E_{f}$ is a closed subset of $Y$.

Proof. Let $y \in \bar{E}_{f}$. Then there is a net $\left\{y_{\alpha}\right\}_{\alpha \in M} \in E_{f}$ such that $y_{\alpha} \rightarrow y$. For every $\alpha \in M$, there is a net $\left\{x_{\beta}^{\alpha}\right\}_{\beta \in M_{\alpha}}$ in $X$ with $x_{\beta}^{\alpha} \rightarrow \infty$ and $f\left(x_{\beta}^{\alpha}\right) \rightarrow y_{\alpha}$. Thus, there is a diagonal net $x_{\beta_{\alpha}}^{\alpha}$ in $X$ with $x_{\beta_{\alpha}}^{\alpha} \rightarrow \infty$ and $f\left(x_{\beta_{\alpha}}^{\alpha}\right) \rightarrow y$. Therefore $y \in E_{f}$ and $E_{f}$ is closed.

2.1.10 Remark. In general, $S E_{f}$ is not closed. For example, let $X=\{a\}$ and $Y=$ $\{1,2\}$ with the indiscrete topology and let $f: X \rightarrow Y$ be defined by $a \rightarrow 1$. Then, $E_{f}=\varnothing$ and $S E_{f}=\{2\}$ which is not closed in $Y$. 
2.1.11 Theorem. [1] Let $Y$ be Hausdorff. Then a continuous mapping $f: X \rightarrow Y$ of $X$ and $Y$ is proper if and only if $E_{f}=\emptyset$.

Proof. If $f$ is proper, by the Bourbaki Theorem, it is easy to show that $E_{f}=\emptyset$. Conversely, assume that $Y$ is Hausdorff and the set $E_{f}=\emptyset$. Take $y \in$ $Y$. Since $Y$ is $T_{2}, f^{-1}(y)$ is closed subset of $X$. Suppose that $f^{-1}(y)$ is not quasicompact. Then there is a net $x_{\alpha} \rightarrow \infty$ in $f^{-1}(y)$ and hence in $X$. So, we have $f\left(x_{\alpha}\right)=y \rightarrow y$ which implies that $y \in E_{f}$, which is a contradiction. To show that the map $f$ is closed. Take $F$ is a closed subset of $X$, and $y \in \overline{f(F)}$. Then there is a net $x_{\alpha}$ in $F$ such that $f\left(x_{\alpha}\right) \in f(F)$ and $f\left(x_{\alpha}\right) \rightarrow y$. Since $E_{f}=\emptyset$, the net $x_{\alpha}$ must have a subnet $x_{\alpha_{\beta}}$ converges to a point in $X$, say $x$. Since $F$ is closed, $x$ is also in $F$. So, by continuity of $f, f\left(x_{\alpha_{\beta}}\right) \rightarrow f(x)$. Since $Y$ is $T_{2}, f(x)=y \in f(F)$. Therefore, $f(F)$ is closed and $f$ is a closed map.

2.1.12 Remark. The $T_{2}$ condition in first direction of the above theorem is not necessarily, however if $Y$ is not $T_{2}$, the second direction is not true. See the example in the remark (21.10), it is obvious that every mapping from $X$ into $Y$ is not proper, since it is not closed. Since $X$ is compact, then $E_{f}=\emptyset$.

2.1.13 Proposition. Every proper map is a quasi-compact map.

Proof. Let $f: X \rightarrow Y$ be a continuous map. Assume that $f$ is proper. Take a quasicompact subset $K$ of $Y$. Suppose that $f^{-1}(K)$ is not quasi-compact, then there is a net $x_{\alpha}$ in $f^{-1}(K)$ such that $x_{\alpha} \rightarrow \infty$. Then a net $f\left(x_{\alpha}\right) \in K$ has convergent subnet, say itself. So, $f\left(x_{\alpha}\right) \rightarrow k \in K$. Since $E_{f}=\emptyset$, this implies that the net $x_{\alpha}$ must have a convergent subnet which is a contradiction.

2.1.14 Theorem. [2] A continuous map $f: X \rightarrow Y$ is proper if and only if $S E_{f}=\emptyset$. 
Proof. Assume that $S E_{f}=\emptyset$. To prove $f$ is closed and quasi compact, if $F$ is a closed subset of $X$ and $y \in \overline{f(F)}$, then there is a net $f\left(x_{\alpha}\right)$ in $f(F)$ converging to $y$. $S E_{f}=\emptyset$ implies $f^{-1}(y) \cap \operatorname{clu}\left(x_{\alpha}\right) \neq \emptyset$. Then there is a point $x \in f^{-1}(y) \cap$ $\operatorname{clu}\left(x_{\alpha}\right)$ and hence there is a subnet $x_{\alpha \beta}$ of $x_{\alpha}$ such that $x_{\alpha \beta} \rightarrow x \in F$. Since $F$ is closed, and $f(x)=y \in f(F)$. Therefore, $f(F)$ is closed. Now, if $K$ is a quasicompact subset of $Y$ and $x_{\alpha}$ is a net in $f^{-1}(K)$, then there is a point $y \in K$ such that $f\left(x_{\alpha}\right) \rightarrow y . S E_{f}=\varnothing$ implies that there is a point $x \in f^{-1}(y) \cap \operatorname{clu}\left(x_{\alpha}\right) \neq \varnothing$. Thus, there is a subnet $x_{\alpha \beta}$ of $x_{\alpha}$ such that $x_{\alpha \beta} \rightarrow x \in f^{-1}(y) \subseteq f^{-1}(K)$. Therefore, $f^{-1}(K)$ is quasi compact. Conversely, assume that $f$ is closed and $f^{-1}(y)$ is quasi compact for every $y \in Y$. Suppose that there is a point $y \in S E_{f}$. Then, there is a net $x_{\alpha} \in X$ such that $f\left(x_{\alpha}\right) \rightarrow y$ and $f^{-1}(y)$ does not meet $\operatorname{clu}\left(x_{\alpha}\right)$. Since $f$ is closed, then $f^{-1}(y) \neq \varnothing$ and a quasi-compact then for every point $z \in f^{-1}(y)$ there is an open set $U_{z}$ containing $z$ and there is $\alpha_{z}$ such that $x_{\alpha} \notin U_{z}$ for each $\alpha>\alpha_{z}$. The collection $\left\{U_{z}\right\}_{z \in f^{-1}(y)}$ forms an open cover to $f^{-1}(y)$, which is quasi compact, there is a finite subcover $\left\{U_{z_{1}}, U_{z_{2}}, U_{z_{3}}, \ldots, U_{z_{n}}\right\}$ and an index $\delta$ such that $x \alpha \notin U=\cup_{i=1}^{n} U_{z_{i}}$ for each $\alpha>\delta$. Put $V=$ $Y \backslash f(X \backslash U) . f$ is closed, and $V$ is open containing $y$. However, $f\left(x_{\alpha}\right) \notin V$ for each $\alpha>\delta$ which contradicts that $f\left(x_{\alpha}\right)$ converges to $y$. Therefore $S E_{f}=\emptyset$.

\subsection{Exceptional Sets in $G$-space Theory}

Recall that if $X$ is $G$-space, we say $G$ acts properly (Bourbaki proper) on $X$ if the map $\theta: G \times X \rightarrow X \times X$ is given by $(g, x) \rightarrow(x, g x)$ is a proper mapping. So, if $\theta$ is proper, then the set $E_{\theta}=\emptyset$. Let us verify the definition of $E_{\theta}$ in $G$-space 
notations in more explicitly. By the definition of exceptional set $E_{\theta}$, it equals the set:

$\left\{(x, y) \in X \times X: \exists\right.$ a net $\left(x_{\alpha}, g_{\alpha}\right) \rightarrow \infty$ in $G \times X$ such that $\left.\left(x_{\alpha}, g_{\alpha} x_{\alpha}\right) \rightarrow(x, y)\right\}$.

Since the net $\left(x_{\alpha}, g_{\alpha} x_{\alpha}\right) \rightarrow(x, y)$, then $x_{\alpha} \rightarrow x$ and $g_{\alpha} x_{\alpha} \rightarrow y$. Also, $\left(x_{\alpha}, g_{\alpha}\right) \rightarrow$ $\infty$ implies to $g_{\alpha} \rightarrow \infty$. We can rewrite the above set as following:

$\left\{(x, y) \in X \times X: \exists\right.$ a net $x_{\alpha} \rightarrow x$ in $X \wedge$ a net $g_{\alpha} \rightarrow \infty$ in $G$ such that $\left.g_{\alpha} x_{\alpha} \rightarrow y\right\}$.

Since the point $x$, the first coordinate of the above set, is determined by a net converges to it. We may put $E_{\theta}=\Lambda(x)$ if $x_{\alpha}=x$ or, in general, $E_{\theta}=J(x)$ if $x_{\alpha} \neq x$. That means the sets $\Lambda(x)$ and $J(x)$ reflect the properties of the set $E_{\theta}$.

2.2.1 Definition. Let $X$ be a $G$-space and $x \in X$. We define the limit set and its prolongation associated with the point $x$ as following:

$J(x)=\left\{y_{\in} X\right.$ : there is a net $\left\{x_{\alpha}\right\}$ in $X$ and a net $\left\{g_{\alpha}\right\}$ in $G$ with $x_{\alpha} \rightarrow$

$x$ and $g_{\alpha} \rightarrow \infty$ such that $\left.g_{\alpha} x_{\alpha} \rightarrow y\right\}$.

$\Lambda(x)=\left\{y_{\in} X:\right.$ there is a net $\left\{g_{\alpha}\right\}$ in $G$ with $g_{\alpha} \rightarrow \infty$ and $\left.g_{\alpha} x \rightarrow y\right\}$.

2.2.2 Proposition. Let $X$ be a $G$-space and $x \in X$. Then,

(i) For every $x$ in $X, \Lambda(x), J(x)$ are invariant sets under $G$.

(ii) If an orbit $G x$ is closed, then $\Lambda(x) \subseteq G x$.

(iii) For every $x$ in $X, \Lambda(x), J(x)$ are closed.

(iv) $\overline{G x} \supseteq G x \cup \Lambda(x)$.

(v) $y \in J(x)$ if and only if $x \in J(y)$.

(vi) If $X$ is the discrete $G$ - space, then $\Lambda(x)=J(x)$ for all $x$ in $X$.

(vii) If $x \in J(x)$, then for each $y \in G x, y \in J(x)$.

(viii) If $y \in J(x)$, then for each $z \in G x, y \in J(z)$. 
(ix) For every $g \in G$ and $x \in X, g \Lambda(x)=\Lambda(g x)=\Lambda(x)$ and

$$
g J(x)=J(g x)=J(x)
$$

Proof. For $i$, let $y \in \Lambda(x)$ and $g \in G$. Then there is a net $\left\{g_{\alpha}\right\}$ in $G$ with $g_{\alpha} \rightarrow \infty$ and $g_{\alpha} x \rightarrow y$. It is clear that $\left\{g g_{\alpha}\right\}$ is a net in $G$ with $g g_{\alpha} \rightarrow \infty$, and by continuity of the action $\left(g g_{\alpha}\right) x \rightarrow g y$ which implies that $g y \in \Lambda(x)$ and hence $\Lambda(x)$ is invariant. The proof for $J(x)$ is similar. For $i i$, let $G x$ is a closed subset of $X$ and $y \in \Lambda(x)$. There is a net $g_{\alpha} \rightarrow \infty$ in $G$ and $g_{\alpha} x \rightarrow y$. Since $G x$ is closed and $g_{\alpha} x \in G x$, then $y \in G x$. For $i i i$. See proposition (2.1.11). For $i v$, the proof is obvious. For $v$, let $y \in J(x)$. Then there is a net $\left\{g_{\alpha}\right\}$ in $G$ with $g_{\alpha} \rightarrow \infty$ and there is a net $\left\{x_{\alpha}\right\}$ in $X$ with $x_{\alpha} \rightarrow x$ such that $g_{\alpha} x_{\alpha} \rightarrow y$. Put $y_{\alpha}=g_{\alpha} x_{\alpha} \rightarrow y$, then it is clear that $g_{\alpha}^{-1} \rightarrow \infty$. We have $g_{\alpha}^{-1} y_{\alpha}=g_{\alpha}^{-1}\left(g_{\alpha} x_{\alpha}\right)=x_{\alpha} \rightarrow x$. Thus $x \in J(y)$. For $v i$, vii, viii proofs are obvious. For $i x$, let $y \in g \Lambda(x)$, and let $y=g z$ for some $z \in \Lambda(x)$. Then there is a net $g_{\alpha} \rightarrow \infty$ such that $g_{\alpha} x \rightarrow z$. By continuity of the action, we have $g g_{\alpha} x \rightarrow g z$ and since $g g_{\alpha} \rightarrow \infty$, we have $y=g z \in \Lambda(x)$. If $y \in$ $\Lambda(x)$, then $g^{-1} y \in \Lambda(x)$ for every $g \in G$ and thus $g\left(g^{-1} y\right) \in g \Lambda(x)$. Therefore, $g \Lambda(x)=\Lambda(x)$ for every $g \in G$. Now, take $y \in \Lambda(x)$, then there is a net $g_{\alpha} \rightarrow \infty$ such that $g_{\alpha} x \rightarrow y$. Since $g_{\alpha}\left(g^{-1} g\right) x \rightarrow y$ and $g_{\alpha} g^{-1} \rightarrow \infty$. Thus, $y \in \Lambda(g x)$. If $y \in \Lambda(g x)$ for some $g \in G$, then there is a net $g_{\alpha} \rightarrow \infty$ such that $g_{\alpha}(g x) \rightarrow y$. $g_{\alpha} g \rightarrow \infty$ implies to $y \in \Lambda(x)$. Therefore, $\Lambda(x)=\Lambda(g x)$ for every $g \in G$. Proofs for part for $J(x)$ proofs are similar. For $i x$, the proof is obvious.

2.2.3 Remark. The second side in $i i$, and the quality in $i v$ is not necessary. For example, if $G=\{-1,1\}$ with the discrete topology acts on the plane with the indiscrete topology. It is obvious that for every $x$ in $X, \Lambda(x)=\emptyset \subset G x=\{x,-x\}$ and $G x$ is not closed subset of $X$. Moreover, $\bar{G}_{x}=X$. However, if $X$ is $T_{2}$ the second side in $i i$ and the quality in $i v$ hold. 
In this stage, we present some results that could be characterized by the above sets.

2.2.4 Theorem. [3] Let $X$ be a $T_{2}$ space, and $G$ acts continuously on $X$. The action is Bourbaki proper if and only if the $J(x)=\emptyset$ for every $x \in X$.

2.2.5 Remark. It is suitable in this place to be referred that for $x \in X$, the set $\theta^{-1}(x, x)=\{(g, y) \in G \times X: \theta(g, y)=(y, g y)=(x, x)\}=\{g \in G: g x=x\}=$ $G_{x} \times\{x\}$.That is an action $G$ on a space $X$ is Bourbaki proper if and only if all stabilizers $G_{x}$ are quasi-compact and the map $\theta: G \times X \rightarrow X \times X,(g, x) \rightarrow(x, g x)$ is closed.

2.2.6 Corollary. The condition $x \notin J(x)$ (without $T_{2}$ ) implies to $\theta^{-1}(x, x)$ be quasi-compact.

2.2.7 Remark. The converse of the above corollary is not true. For example, an action on $\mathbb{R}^{2} \backslash\{0\}$ by the discrete topological group $G=\mathbb{R}$, the group of the real numbers, is given by $t(x, y)=\left(x e^{t}, y e^{t}\right)$. In such $G$-space we have for every point $z=(x, y)$ in the plane $\theta^{-1}(z, z)=\{0\} \times\{z\}$ which is quasi-compact.

However, $(x, y) \in J((x, y))$. Take $t_{n}=\frac{1}{n}$, then for every $(x, y)$ in the plane we have $t_{n}(x, y) \rightarrow(x, y)$.

2.2.8 Corollary. If $y=g x$ for some $g \in G$, The condition $x \notin J(x)$ (without $T_{2}$ ) implies to $\theta^{-1}(x, y)$ is quasi-compact.

Proof. It is obvious and comes from property vii.

2.2.9 Proposition. If the orbit space $X / G$ is $T_{2}$, then for every pair of points $x, y \in$ $X$ such that $\tilde{x} \neq \tilde{y}$, and $y \notin J(x)$. Moreover, if $X$ is $T_{2}$, the converse is also true.

Proof. Assume that the orbit space $X / G$ is $T_{2}$ and $x, y \in X$ such that they satisfy as above. To show that $y \notin J(x)$. Suppose that $y \in J(x)$, then these a net $g_{\alpha} \rightarrow \infty$ in $G$ and a net $x_{\alpha} \rightarrow x$ in $X$ such that $g_{\alpha} x_{\alpha} \rightarrow y$. Take $U_{x}$ a neighborhood of $x$ and $V_{y}$ a neighborhood of $y$. There is a $\delta \in D$, such that $x_{\alpha} \in U_{x}$ and $g_{\alpha} x_{\alpha} \in$ 
$V_{y}$ for every $\alpha \geq \delta$. That is $g_{\alpha} \in\left(\left(U_{x}, V_{y}\right)\right)$ for every $\alpha \geq \delta$, this implies to $\widetilde{U}_{x} \cap$ $\tilde{V}_{y} \neq \emptyset$. Since $U_{x}$ and $V_{y}$ are arbitrary neighborhoods of $x$ and $y$, we have that the orbit space is not $T_{2}$ which is a contradiction. Therefore, $y \notin J(x)$. Now assume that $X$ is $T_{2}$, and every pair of points $x, y \in X$ with $y \neq g x$ for every $g \in G$. Suppose that the orbit space is not $T_{2}$. Then is a pair of points $\tilde{x} \neq \tilde{y}$ of $X / G$ such that there are not disjoint open sets in $X / G$ separate them. So, every pair of neighborhoods $N_{x}$ and $N_{y}$ of $x \in \tilde{x}$ and $y \in \tilde{y}$, then $\bar{N}_{x} \cap \bar{N}_{y} \neq \emptyset$. We can construct a net $\tilde{x}_{\alpha}$ in $X / G$ such that for every $\alpha \in D, \tilde{x}_{\alpha} \in \widetilde{N}_{x} \cap \widetilde{N}_{y}$. Moreover, $\tilde{x}_{\alpha} \rightarrow \bar{x}$ and $\tilde{x}_{\alpha} \rightarrow \tilde{y}$. Then we find nets $z_{\alpha} \in \tilde{x}_{\alpha}$ and $g_{\alpha} z_{\alpha} \in \tilde{x}_{\alpha}$ such that $z_{\alpha} \rightarrow$ $z \in \tilde{x}$ and $g_{\alpha} z_{\alpha} \rightarrow w \in \tilde{y}$. Since $y \notin J(x)$, then $w \notin J(z)$. So $g_{\alpha} \rightarrow$ $g$ for some $g \in G$. The continuity of the action implies to $g_{\alpha} z_{\alpha} \rightarrow g z$ and $w$. By $T_{2}$ of $X$ then we have $g z=w$ which contradicts $\tilde{z}=\tilde{x} \neq \tilde{y}=\widetilde{w}$. Therefore $X / G$ is $T_{2}$.

2.2.10 Remark. If $X$ is not $T_{2}$, the converse is not true in general (see the example in the remark (2.2.3)).

2.2.11 Proposition. [3] For a $G$-space $X$ and a point $x \in X, x \notin \Lambda(x)$ implies to the stabilizer subgroup $G_{x}$ of $G$ is quasi-compact.

2.2.12 Remark. In general, if the stabilizer of a point $x$ is quasi-compact, then it is not necessarily that $x \notin \Lambda(x)$, see the example in the remark (2.2.7).

2.2.13 Proposition. [3] If $G$ is non-compact, then $x \notin \Lambda(x)$ implies to that $x$ is not periodic point [ $\cong G_{x} K \neq G$ for every compact subset $K$ of $\left.G\right]$. 


\subsection{Strongly Exceptional Sets in $G$-space}

Let $X$ be a $G$-space and let $G$ act on $X$ properly. Then, the map $\theta: G \times X \rightarrow$ $X \times X$ such that $(g, x) \rightarrow(x, g x)$ is proper in the sense of Bourbaki. So, $\theta$ is proper if and only if the set $S E_{\theta}=\emptyset$. Again, let us verify the definition of $S E_{\theta}$ in $G$-spaces notations. By the definition of the strongly exceptional set $S E_{\theta}$, it equals to the set: $\left\{(x, y) \in X \times X: \exists\right.$ a net $\left(g_{\alpha}, x_{\alpha}\right)$ in $G \times X$ such that $\left(x_{\alpha}, g_{\alpha} x_{\alpha}\right) \rightarrow$ $(x, y)$ and $\left.\operatorname{clu}\left(\left(g_{\alpha}, x_{\alpha}\right)\right) \cap \theta^{-1}(x, y)=\emptyset\right\}$. Since the net $\left(x_{\alpha}, g_{\alpha} x_{\alpha}\right) \rightarrow$ $(x, y)$, then $x_{\alpha} \rightarrow x$ and $g_{\alpha} x_{\alpha} \rightarrow y$. Also, $\operatorname{clu}\left(\left(g_{\alpha}, x_{\alpha}\right)\right) \cap \theta^{-1}(x, y)=\emptyset$ implies to either $\operatorname{clu}\left(g_{\alpha}\right) \cap((\{x\},\{y\}))=\emptyset$ or $\operatorname{clu}\left(x_{\alpha}\right) \cap\{x\}=\emptyset$. Since $x_{\alpha} \rightarrow x$, we can rewrite the above set as following:

$\left\{(x, y) \in X \times X: \exists\right.$ a net $x_{\alpha} \rightarrow x$ in $X \wedge$ a net $g_{\alpha}$ in $G$ such that $g_{\alpha} x_{\alpha} \rightarrow y \wedge$ $\left.\operatorname{clu}\left(g_{\alpha}\right) \cap((\{x\},\{y\}))=\emptyset\right\}$. Since the point $x$, the first coordinate of the above set, is determined by a net converges to it. We may put $S E_{\theta}=\Lambda_{S}(x)$ if $x_{\alpha}=x$ or, in general, $S E_{\theta}=J_{S}(x)$ if $x_{\alpha} \neq x$. That means the sets $\Lambda_{S}(x)$ and $J_{S}(x)$ reflect the properties the set $S E_{\theta}$.

2.3.1 Remark. It is obvious that for every $x \in X$ then $\Lambda(\mathrm{x}) \subseteq \Lambda_{S}(x)$ and $J(x) \subseteq$ $J_{S}(x)$ and if $X$ is $T_{2}$, then we have $\Lambda(\mathrm{x})=\Lambda_{S}(x)$ and $J(x)=J_{S}(x)$.

We give some properties for these sets in an arbitrary $G$-space $X$.

2.3.2 Proposition. Let $X$ be a $G$-space and $x \in X$. Then

1. $J_{S}(x), \Lambda_{S}(x)$ are invariant.

2. $\overline{G x}=G x \cup \Lambda_{S}(x)$.

3. $G x$ is closed if and only if $\Lambda_{S}(x) \subseteq G x$.

4. If $y \in J_{S}(x)$ then $x \in J_{S}(y)$.

5. For every $g \in G$, then $g \Lambda_{S}(x)=\Lambda_{S}(g x)=\Lambda_{S}(x)$.

6. For every $g \in G$, then $g J_{S}(x)=J_{S}(g x)=J_{S}(x)$. 
7. If $y \in J_{S}(x)$ then $y \in J_{S}(z)$ for every $z \in G x$.

8. If $y \in J_{S}(x)$ then $z \in J_{S}(x)$ for every $z \in G y$.

Proof. For 1, we shall show proof for the set $J_{S}(x)$ and the proof for $\Lambda_{S}(x)$ is similar. For invariance of $J_{S}(x)$, take $g \in G$ and $y \in J_{S}(x)$. Then there is a net $x_{\alpha} \rightarrow x$ in $X$ and a net $g_{\alpha}$ in $G$ such that $g_{\alpha} x_{\alpha} \rightarrow y$ and $\operatorname{clu}\left(g_{\alpha}\right) \cap((\{x\},\{y\}))=$ $\emptyset$. Since $g g_{\alpha} x_{\alpha} \rightarrow g y$ and $g g_{\alpha}$ is a net in $G$, then it suffices to show that $\operatorname{clu}\left(g g_{\alpha}\right) \cap((\{x\},\{g y\}))=\varnothing$. Suppose that there is a point $g_{1} \in \operatorname{clu}\left(g g_{\alpha}\right) \cap$ $((\{x\},\{g y\}))$, then there is a subnet $g g_{\alpha_{\beta}}$ of the net $g g_{\alpha}$ in $G$ such that $g g_{\alpha_{\beta}} \rightarrow g_{1}$ and $g_{1} x=g y$. Then we have $g_{\alpha_{\beta}} \rightarrow g^{-1} g_{1}$ and $g^{-1} g_{1} x=y$. Thus $g^{-1} g_{1} \in$ $\operatorname{clu}\left(g_{\alpha_{\beta}}\right) \cap((\{x\},\{y\})) \subseteq \operatorname{cl}\left(g_{\alpha}\right) \cap((\{x\},\{y\}))=\emptyset$ which is a contradiction. Therefore, $\operatorname{clu}\left(g g_{\alpha}\right) \cap((\{x\},\{g y\}))=\emptyset$ and $g y \in J_{S}(\mathrm{x})$. For 2 , it is obvious that $G x \cup \Lambda_{S}(x) \subseteq \overline{G x}$. Conversely, take $y \in \overline{G x}$. Then there is a net $y_{\alpha}=g_{\alpha} x \in G x$ and $g_{\alpha} x \rightarrow y$. Either $g_{\alpha} \rightarrow \infty$, then $y \in \Lambda(x) \subseteq \Lambda_{S}(x)$ or $g_{\alpha}$ has a cluster point $g_{1}$, then if $g_{1} x=y \in G x$ and we are done. Otherwise, $\operatorname{clu}\left(g_{\alpha}\right) \cap((\{x\},\{y\}))=\emptyset$. Thus, $y \in \Lambda_{S}(x)$. For 3 , if $G x$ is closed, then $\overline{G x}=G x$. By 2 , we have $\Lambda_{S}(x) \subseteq G x$. On the other hand, if $\Lambda_{S}(x) \subseteq G x$, then also by $2, \overline{G x}=G x \cup \Lambda_{S}(x)=G x$. Therefore, $G x$ is closed. For 4, if $y \in J_{S}(x)$, there is a net $x_{\alpha} \rightarrow x$ in $X$ and a net $g_{\alpha}$ in $G$ such that $g_{\alpha} x_{\alpha} \rightarrow y$ and $\operatorname{clu}\left(g_{\alpha}\right) \cap((\{x\},\{y\}))=\emptyset$. Put $y_{\alpha}=g_{\alpha} x_{\alpha}$. Then $y_{\alpha} \rightarrow y$ is a net in $X$ and $g_{\alpha}^{-1}$ is a net in $G$ such that $g_{\alpha}^{-1} y_{\alpha} \rightarrow x$. It suffices to show that $\operatorname{clu}\left(g_{\alpha}^{-1}\right) \cap((\{y\},\{x\}))=\emptyset$. Suppose that there is $g \in \operatorname{clu}\left(g_{\alpha}^{-1}\right) \cap$ $((\{y\},\{x\}))$. Then there is a subnet $g_{\alpha_{\beta}}^{-1}$ of $g_{\alpha}^{-1}$ with $g_{\alpha_{\beta}}^{-1} \rightarrow g$ and $g y=x$. Thus, $g_{\alpha_{\beta}} \rightarrow g^{-1}$ and $g^{-1} x=y$. By continuity of the action, we have $g_{\alpha_{\beta}} x_{\alpha} \rightarrow g^{-1} x=$ $y$. Thus, $g^{-1} \in \operatorname{clu}\left(g_{\alpha_{\beta}}\right) \cap((\{x\},\{y\})) \subseteq \operatorname{clu}\left(g_{\alpha}\right) \cap((\{x\},\{y\}))=\emptyset$ which is a contradiction. Therefore, $\operatorname{clu}\left(g_{\alpha}^{-1}\right) \cap((\{y\},\{x\}))=\emptyset$ and $x \in J_{S}(y)$. The proofs for 7 , and 8 come immediately from the property in 1 , and the proof in 5 is a 
special case in 6 . For 6 , let $z \in g J_{S}(x)$, and let $z=g y$ for some $y \in J_{S}(x)$. Then there is a net $x_{\alpha} \rightarrow x$ in $X$ and a net $g_{\alpha}$ in $G$ such that $g_{\alpha} x_{\alpha} \rightarrow y$ and $\operatorname{clu}\left(g_{\alpha}\right) \cap$ $((\{x\},\{y\}))=\emptyset$. Consider a net $g_{\alpha} g^{-1}$ in $G$. It is obvious that $\left(g_{\alpha} g^{-1}\right) g x_{\alpha} \rightarrow y$ and $g x_{\alpha} \rightarrow g x$. We want to show that $\operatorname{clu}\left(g_{\alpha} g^{-1}\right) \cap((\{g x\},\{y\}))=\emptyset$. Suppose there is $g_{1} \in \operatorname{clu}\left(g_{\alpha} g^{-1}\right) \cap((\{g x\},\{y\}))$. Then there is a subnet $g_{\alpha_{\beta}} g^{-1}$ of $g_{\alpha} g^{-1}$ with $g_{\alpha_{\beta}} g^{-1} \rightarrow g_{1}$ and $g_{1} g x=y$. We have $g_{\alpha_{\beta}} \rightarrow g_{1} g$, and then $g_{\alpha_{\beta}} x_{\alpha} \rightarrow g_{1} g x=y$. Thus, $g_{1} g \in \operatorname{clu}\left(g_{\alpha_{\beta}}\right) \cap((\{x\} .\{y\})) \subseteq \operatorname{clu}\left(g_{\alpha}\right) \cap$ $((\{x\},\{y\}))=\emptyset$. Which is a contradiction. Therefore, $y \in J_{S}(g x)$ and by $1, g y=$ $z \in J_{S}(g x)$. By 1 , it is obvious that $J_{S}(g x)=J_{S}(x)$. We shall show that $J_{S}(x) \subseteq$ $g J_{S}(x)$. Take $z \in J_{S}(x)$. Then $g^{-1} z \in J_{S}(x)$ and again $z=g g^{-1} z \in g J_{S}(x)$.

2.3.3 Theorem. [ Main Theorem] Let $X$ be a $G$-space. Then $G$ acts properly on $X$ if and only if for every point $x \in X$, the set $J_{S}(x)=\emptyset$.

Proof. The proof comes immediately from the theorem (2.1.14) and from the fact that $J_{S}(x)=S E_{\theta}$.

2.3.4 Remark. As result that Bourbaki proper implies that the orbit spaces are $T_{2}$. 2.3.5 Corollary. Let $X$ be a $G$-space. If $J_{S}(x)=\emptyset$ for every $x \in X$, then the orbit space $X / G$ is Hausdorff.

Proof. Let $\tilde{x}, \tilde{y}$ be two distinct points in $X / G$. Suppose that every pair of open neighborhoods $U_{\bar{x}}, V_{\bar{y}}$ of $\tilde{x}, \tilde{y}$ respectively do not separate them. Then we may construct a net from those intersections for every pair. That is, there is a net $\tilde{x}_{\alpha} \in$ $U_{\alpha} \cap V_{\alpha} \neq \varnothing$ such that $\tilde{x}_{\alpha}$ converges to both $\tilde{x}, \tilde{y}$. Put $x_{\alpha}^{1} \in \pi^{-1}\left(\tilde{x}_{\alpha}\right)$ and $x_{\alpha}^{2} \in$ $\pi^{-1}\left(\tilde{x}_{\alpha}\right)$ such that $x_{\alpha}^{1} \rightarrow x \in \pi^{-1}(\tilde{x})$ and $x_{\alpha}^{2} \rightarrow y \in \pi^{-1}(\tilde{y})$ and $g_{\alpha} x_{\alpha}^{1}=x_{\alpha}^{2}$. Since $J_{S}(x)=\varnothing$, then $\operatorname{clu}\left(g_{\alpha}\right) \cap((\{x\},\{y\})) \neq \varnothing$ which contradicts that $\tilde{x} \neq \tilde{y}$.

Therefore $X / G$ is Hausdorff. 


\subsection{Limit Sets and Proper actions}

In this section, we shall present some interesting results using the limit set and its prolongation set in their proofs as convergence technique and also, we show how such these sets could be good keys to describe the behaviors of orbits in orbit spaces. First, we recall some well-known results have proved by limit sets under certain conditions. Second, we present more results on $G$-space notions under weaker conditions using our keys the sets $\Lambda(x)$ and $\Lambda_{S}(x), J(x)$ and $J_{S}(x)$. In a compact ( $T_{2}$ axiom included) topological group acts on a Hausdorff space, there is concert relations between points and their stabilizers [9]. We shall review these relations and then we give our proofs under weaker conditions. Finally, we give a new proof for a well-known theorem “Conjugacy Neighborhoods Theorem”.

2.4.1 Remark. From now on, a setting where $G$ is a locally compact ( $T_{2}$ is included) acting on a completely regular space $X$ is called a Palais setting (or setting in the sense of Palais). In the rest, whenever we mention Cartan or Palais proper $G$-space that we mean certainly $G$ and $X$ endowed with these properties in. this setting.

2.4.2 Proposition. [13] Let $X$ be a $G$-space. $X$ is Cartan $G$-space (in the sense of Palais) iff $x \notin J(\mathrm{x})$ for each $x \in X$.

2.4.3 Proposition. [26] Let $X$ be a $G$-space, where $X$ and $G$ are merely Hausdorff. Then $X$ is Bourbaki proper $G$-space if and only if $J(x)=\phi$ for each $x \in X$.

Also, the author, in [3], has given several properties of limits sets when $G$ is a locally compact and $X$ is a completely regular topological space and their relations with small and thin notions. The following results in [3] could be proved in certain weaker conditions. 
2.4.4 Proposition. Let $X$ be a $T_{2} G$-space and $x \in X$. Then the orbit space $X / G$ is $T_{2}$ iff for each $x \in X, J(x)$ is a subset of $G x$.

Proof. If $X / G$ is $T_{2}$, then for every pair $\bar{x}$ and $\bar{y}$ in $X / G$ with $\bar{x} \neq \bar{y}$ we have $y \notin$ $J(x)$. For an arbitrary point $x \in X$, if $x \notin J(x)$, then $J(x)=\emptyset \subseteq G x$. Otherwise, that is $x \in J(x)$, then by inveracity of $J(x)$ we have $G x \subseteq J(x)$. From the first step of the proof we have shown that for every $y \neq g x$ for some $g \in G, y \notin J(x)$. Thus $J(x)$ is one component, that is $J(x)=G x$. Conversely, let $J(x) \subseteq G x$ and suppose that the orbit space is not $T_{2}$. Then there is a pair of two distinct points $\bar{x}$ and $\bar{y}$ in $X / G$ such that they could not be separated by two disjoint open neighborhoods in $X / G$. Then we can construct a net $\bar{x}_{\alpha}$ in $X / G$ such that $\bar{x}_{\alpha} \rightarrow \bar{x}$ and $\bar{x}_{\alpha} \rightarrow \bar{y}$. Then there are nets $x_{\alpha}^{1}, x_{\alpha}^{2}$ in $\bar{x}_{\alpha}$ and a net $g_{\alpha}$ in $G$ such that $x_{\alpha}^{1} \rightarrow x, x_{\alpha}^{2} \rightarrow y$ and $g_{\alpha} x_{\alpha}^{1}=$ $x_{\alpha}^{2}$. Either $g_{\alpha} \rightarrow \infty$ or $g_{\alpha} \rightarrow g$ for some $g \in G$. For the first case we have $y \in$ $J(x) \subseteq G x$ and in the other we have $g_{\alpha} x_{\alpha}^{1}=x_{\alpha}^{2} \rightarrow g x=y$ where the quality is hold if $X$ is $T_{2}$. Thus, both cases implies to a contradiction. Therefore, $X / G$ is $T_{2}$.

2.4.5 Proposition. [3] Let $X$ be a Cartan $G$-space. Then for each $x \in X, \Lambda(x)=$ $\emptyset$.

2.4.6 Proposition. Let $G$ be a locally quasi-compact group acting on a $T_{2}$ space $X$ and let $x \in X$. Then $x \notin J(x)$ if and only if there is a neighborhood $U$ of $x$ and a compact neighborhood $V$ of $e, e$ is the identity element of $G$, such that $g U \cap U=$ $\emptyset$ for each $g \notin V$.

Proof. If $G$ is quasi-compact, the proof is trivial since then $J(x)=\emptyset$ for every $x \in$ $X$ and so one could take $V=G$ for the condition in the proposition. Let $G$ be an arbitrary locally quasi-compact. First, we assume that $x \notin J(x)$ for some $x \in X$. Suppose that if $U$ is a neighborhood of $x$ and $V$ is a quasi-compact of the identity, 
then there is a $g \notin V$ and $g U \cap U \neq \varnothing$. Let $\left\{U_{\alpha}\right\}$ be the neighborhood system of the point $x$. Then for every $\alpha$ there a point $g_{\alpha} \notin V$ and a point $x_{\alpha} \in U_{\alpha}$ such that $g_{\alpha} x_{\alpha} \in U_{\alpha}$. Since $x_{\alpha} \rightarrow x$ and $g_{\alpha} x_{\alpha} \rightarrow x$ and $x \notin J(x)$, there is a $g \in G$ such that $g_{\alpha} \rightarrow g$. By the continuity of the action of $G$ and $X$ is $T_{2}$, we have $g_{\alpha} x_{\alpha} \rightarrow g x=x$. There is no loss of generality if we assume that $V$ be a neighborhood of the stabilizer $G_{x}$ of $x$ (actually, it is because $G_{x}$ is quasi-compact when $x \notin J(x)$ ). Thus, the net $g_{\alpha}$ is eventually in $V$ which contradicts that $g_{\alpha} \notin V$ for every $\alpha$. Therefore, the condition of the proposition is true. For the other direction, suppose that $x \in J(x)$ for some point $x \in X$. There is a net $x_{\alpha} \rightarrow x$ in $X$ and a net $g_{\alpha} \rightarrow \infty$ in $G$ such that $g_{\alpha} x_{\alpha} \rightarrow x$. By the condition, there is a neighborhood $U$ of $x$ and a quasi-compact $V$ of the identity such that $((U, U)) \subseteq V$. Since $x_{\alpha}$ and $g_{\alpha} x_{\alpha}$ are eventually in $U$. We have $g_{\alpha} \in((U, U)) \subseteq V . V$ is a quasi-compact, then $g_{\alpha}$ must have a convergent subnet which is a contradiction. Therefore, $x \notin J(x)$.

Note. We could also prove the proposition (2.4.6) without $T_{2}$ condition on the space $X$ using only the set $J_{S}(x)$.

2.4.7 Corollary. Let $X$ be a $G$-space as in the above proposition and $x \in X$. Then $x \in J(x)$ if and only if $x$ has no thin neighborhood.

Proof. Assume that $x \in J(x)$ for some $x \in X$. Suppose there is a thin neighborhood $U$ of $x$. Then the closure of the set $((U, U))$ is quasi-compact. $x \in J(x)$ implies that there is a net $x_{\alpha} \rightarrow x$ in $X$ and a net $g_{\alpha} \rightarrow \infty$ in $G$ such that $g_{\alpha} x_{\alpha} \rightarrow x$. Thus, $g_{\alpha} \in$ $((U, U))$ and $g_{\alpha}$ must have convergent subnet which contradiction. Conversely, assume that a point $x \in X$ has no thin neighborhood. Suppose that $x \notin J(x)$. Then by proposition (2.4.6), there is a neighborhood $U$ of $x$ and a quasi-compact $V$ of the identity $((U, U)) \subseteq V$. Since $V$ is a quasi-compact, the its closure $\bar{V}$ is also a quasi- 
compact. Thus, $\overline{((U, U))} \subseteq \bar{V}$. Therefore, $U$ is a thin neighborhood of $x$ which contradicts our assumption.

2.4.8 Remark. By corollary (2.2.8), the condition $y \notin J(x)$ implies to that a set $((\{x\},\{y\}))$ is a quasi-compact. Then we can put the following criterion.

2.4.9 Proposition. Let $G$ be a locally quasi-compact group acting on $T_{2}$ space $X$ and let $x, y$ be a pair of points in $X . y \notin J(x)$ if and only if there is a neighborhood $U$ of $x$ and a neighborhood $W$ of $y$ and a quasi-compact neighborhood $V$ of the $((\{x\},\{y\}))$ such that $g U \cap W=\varnothing$ if $g \notin V$.

Proof. The proof is obvious if $G$ is a quasi-compact. We assume that $G$ be an arbitrary locally quasi-compact. Take a pair of points $x, y$ in $X$ such that $y \notin J(x)$. Suppose that if $U, W$ and $V$ are neighborhood of $x, y$ and $((\{x\} .\{y\}))$ respectively, then there is a $g \notin V$ and $g U \cap W \neq \emptyset$. Let $\left\{U_{\alpha}\right\}$ and $\left\{W_{\alpha}\right\}$ be the neighborhood systems for $x$ and $y$ and let $V$ be a quasi-compact of the identity. Then for every $\alpha$ there is a $g_{\alpha} \notin V$ and $g_{\alpha} U_{\alpha} \cap W_{\alpha} \neq \emptyset$. Thus, for every $\alpha$ there is $x_{\alpha} \in U_{\alpha}$ such that $g_{\alpha} x_{\alpha} \in W_{\alpha}$. Since $x_{\alpha} \rightarrow x$ and $g_{\alpha} x_{\alpha} \rightarrow y$ and $y \notin J(x)$, there is a $g \in G$ such that $g_{\alpha} \rightarrow g$. By the continuity of the action of $G$ and $T_{2}$ property of $X$, we have $g_{\alpha} x_{\alpha} \rightarrow$ $g x=y$ and $g \in((\{x\},\{y\})) \subseteq V . V$ is a neighborhood of $g$, then the net $g_{\alpha}$ is eventually in $V$ which contradicts $g_{\alpha} \notin V$ for every $\alpha$. Conversely, Suppose that $y \in J(x)$. Then there is a net $x_{\alpha} \rightarrow x$ in $X$ and a net $g_{\alpha} \rightarrow \infty$ in $G$ such that $g_{\alpha} x_{\alpha} \rightarrow$ $y$. By hypothesis, there is a neighborhood $U_{x}$ of $x$ and a neighborhood $W_{y}$ of $y$ and $V$ is a quasi-compact neighborhood of $((\{x\},\{y\}))$ such that $\left(\left(U_{x}, W_{y}\right)\right) \subseteq V$. Then, the nets $x_{\alpha}$ and $g_{\alpha} x_{\alpha}$ are eventually in $U_{x}$ and $W_{y}$ respectively and $g_{\alpha} \in$ $\left(\left(U_{x}, W_{y}\right)\right) \subseteq V$. That is, $g_{\alpha}$ must have a convergent subnet which is impossible. Therefore, $y \notin J(x)$. 
Note. In the above proposition we may drop the $T_{2}$ condition on the space $X$, however, we must use the set $J_{s}(x)$ instead of the set $J(x)$.

2.4.10 Corollary. Let $X$ be a $G$-space. For each pair of points $x, y$ in $X$ there is a neighborhood $U$ of $x$ and a neighborhood $W$ of $y$ and there is a quasi-compact neighborhood $V$ of $((\{x\},\{y\}))$ such that $g U \cap W=\emptyset$ for each $g \notin V$ if and only if $J(x)=\emptyset$ for each $x \in X$.

Proof. The proof immediately comes from proposition (2.4.9).

2.4.11 Proposition. Let $X$ be a $G$-space and $y$ be a point in $X$. Then $y$ has no small neighborhood whenever $y \in J(x)$ for some point $x \in X$.

Proof. Let $y \in J(x)$ for some $x, y \in X$. If $S$ is a small neighborhood of $\mathrm{y}$, there is a neighborhood $U$ of $x$ such that a set $((U, S))$ has a quasi-compact closure. Since $y \in$ $J(x)$, there is a net $x_{\alpha} \rightarrow x$ in $X$ and a net $g_{\alpha} \rightarrow \infty$ in $G$ such that $g_{\alpha} x_{\alpha} \rightarrow y$. Thus, $g_{\alpha} \in((U, S))$ which is a quasi-compact which implies to a contradiction since $g_{\alpha} \rightarrow$ $\infty$.

2.4.12 Proposition. Let $G$ be a locally quasi-compact group acting on $T_{2}$ space $X$. Then $J(x)=\emptyset$ for each $x \in X$ if and only if every pair of points of $X$ has relatively thin neighborhoods.

Proof. For the first side, for every pair of points $x, y \in X$. Since $y \notin J(x)=\emptyset$, then by proposition (2.4.9), there are neighborhoods $U_{x}, W_{y}$, and a quasi-compact neighborhood $V$ satisfy the condition in the proposition. That is, $\left(\left(U_{x}, W_{y}\right)\right) \subseteq V$. Since $\bar{V}$ is still quasi-compact, then $\overline{\left(\left(U_{x}, W_{y}\right)\right)}$ is quasi-compact. Therefore, $x, y$ have relatively thin neighborhoods. Conversely, for a pair of points $x, y \in X$, Take $V=\overline{((U, W))}$ where $U$ and $V$ relatively thin neighborhoods of $x$ and $y$ respectively. We have $((U, W)) \subseteq V$ and $V$ is a quasi-compact neighborhood of a 
set $((\{x\},\{y\}))$. By proposition (2.2.10), then $y \notin J(x)$. Since the points $x$ and $y$ are an arbitrary. Therefore, $J(x)=\emptyset$ for every $x \in X$.

2.4.13 Remark. In general, if for every pair of points of $X$, there are relatively thin neighborhoods then for each $x \in X, J(x)=\emptyset$. The converse is not in general. For example, the natural action of the group of rational numbers on itself. Also, if for every pair of points of $X$, there are relatively thin neighborhoods, it does not imply to that $X$ is Palais proper. For instance, see the dynamical system in [7, pp. 46]. However, if $X$ is a locally compact, the notions of Bourbaki and Palais proper are equivalent.

2.4.14 Theorem. [3] Let $X$ be a locally compact $G$-space. Under Palais setting, Palais proper and Bourbaki proper are equivalent.

If $G$ is a compact Lie group acting on $T_{2}$ space, there is a relation between close points and their stabilizers and there is also a relation between close orbits and their kernels. One can show those interesting results in the version of compact Lie group in Montgomery and Zippin [23] and [12]. Palais also has given analogous results for more wider classes of $G$-spaces are called locally proper $G$-spaces (or Cartan $G$ spaces) when $G$ is an arbitrary $T_{2}$-locally compact (Lie) group acting continuously on completely regular space. In the next, we will review those results in both versions and then we shall give our proofs for the same propositions to get the same results using this convergence technique. We merely need the following definition.

2.4.15 Definition. [10] Let $G$ be a topological group acting continuously on a space $X$. and let $x$ be a point in $X$. The kernel of the action $G$ on $X$ is the subgroup $\cap_{x \in X} G_{x}$ and the kernel of action of $G$ on the orbit $G x$ is the subgroup $\cap_{y \in G x} G_{y}$. 
2.4.16 Lemma. [9] (Close points have close stabilizers) Let $G$ acts properly on a space $X$, choose $x \in X$ and let $U$ be a neighborhood of the stabilizers $G_{x}$ of $x$ in $G$. Then $x$ has a neighborhood $V$ such that for every $y \in V$, then $G_{y} \subseteq U$.

2.4.17 Proposition. Let $G$ be an arbitrary topological group acting on $T_{2}$ space $X$. If $x \notin J(x)$ for some point $x \in X$. Then for every neighborhood $U$ of the stabilizer $G_{x}$ of $x$ in $G$ there is a neighborhood $V$ of $x$ such that for every $y \in V$, then $G_{y} \subseteq$ $U$.

Proof. Suppose that the result is not true. That is, there is a neighborhood $U$ of the stabilizer $G_{x}$ of $x$ in $G$ such that for every neighborhood $V$ of $x$, there is a point $y \in V$ such that $G_{y} \not \subset U$. Let $\left\{V_{\alpha}\right\}$ be a neighborhoods system of the point $x$. Then for every $V_{\alpha}$ there is a point $x_{\alpha} \in V_{\alpha}$ such that there is a $g_{\alpha} \in G_{x_{\alpha}}$ and $g_{\alpha} \notin U$. Let $x_{\alpha}$ be the net constructed by our choice of the family $\left\{V_{\alpha}\right\}$, then the net $x_{\alpha} \rightarrow x$. Since $g_{\alpha} x_{\alpha}=x_{\alpha} \rightarrow x$ and $x \notin J(x)$. We have $g_{\alpha} \rightarrow g$ for some $g \in G$. The continuity of the action gives $g_{\alpha} x_{\alpha} \rightarrow g x$ and the $T_{2}$ property on $X$ gives $g x=x$. Thus, $g \in G_{x} \subseteq U$. Then $g_{\alpha} \in U$ for every $\alpha>\alpha$ ofor some $\alpha$ owhich contradicts our assumption. Therefore, the result is true.

2.4.18 Lemma. [9] (Close orbits have close kernels). Let $G$ be a $T_{2}$ group acting properly on a space $X$, choose a point $x \in X$, and let $U$ be a neighborhood of the kernel of the action $G$ on the orbit $G x$. Then $x$ has a neighborhood $V$ such that $U$ contains all kernels which correspond to orbits of points in $V$.

2.4.19 Proposition. Let $G$ be an arbitrary topological group acting on $T_{2}$ space $X$, let $x$ in $X$ and $x \notin J(x)$. Then for every neighborhood $U$ of the kernel of the action $G$ on the orbit $G x, x$ has a neighborhood $V$ such that the kernels of the action of $G$ on all orbits through points in $V$ contained in $U$.

Proof. Suppose that the result is not true, and there is a neighborhood $U$ of the kernel of the action $G$ on $G x$ such that every neighborhood $V$ of $x$ there a point 
$y \in V$ with $\bigcap_{g \in G} G_{g y} \not \subset U$. Let $\left\{V_{\alpha}\right\}$ be a neighborhoods system of the point $x$. Then for every $V_{\alpha}$ there is a point $x_{\alpha} \in V_{\alpha}$ such that there is a $g_{\alpha} \in \bigcap_{g \in G} G_{g x_{\alpha}}$ and $g_{\alpha} \notin U$. Let $x_{\alpha}$ be the net constructed by our choice of the family $\left\{V_{\alpha}\right\}$, then net $x_{\alpha} \rightarrow x$ and let $g \in G$. Then $g x_{\alpha} \rightarrow g x$. Since $g_{\alpha}\left(g x_{\alpha}\right)=g x_{\alpha} \rightarrow g x$ and $g x \notin$ $J(g x)$. We have $g_{\alpha} \rightarrow g_{1}$ for some $g_{1} \in G$. The continuity of the action implies to $g_{\alpha}\left(g x_{\alpha}\right) \rightarrow g_{1}(g x)=g x$ and $g_{1} \in G_{g x}$ for every $g \in G$. Thus $g_{1} \in$ $\cap_{g \in G} G_{g x_{\alpha}} \subseteq U$ and $g_{\alpha} \in U$ eventually which is a contradiction. Therefore, the result is true.

Note. Propositions (2.4.17) and (2.4.19), we may drop the $T_{2}$ condition on the space $\mathrm{X}$ using the condition $x \notin J_{S}(x)$ instead of $x \notin J(x)$.

According to the proposition (2.4.17), and the conjugacy theorem via Zippin and Montgomery [23], if $G$ is a Lie group, then all stabilizer subgroups of points close enough to the point $x$ are conjugate to subgroups of $G_{x}$. Palais has given a direct proof for this theorem using the notion of slices [29]. We will apply convergence techniques to obtain the same result but before taking up the proof, we need some of the following background.

2.4.20 Definition. [16] A locally compact topological group is called having a property $(\boldsymbol{S})$ provided for every $x \neq e$ in a sufficiently small neighborhood $U$ there exists an integer $n$ so that $x^{2^{n}} \notin U$.

2.4.21 Theorem. [16] A locally compact group has a property $(\boldsymbol{S})$ if it has no small subgroup.

2.4.22 Remark. Every Lie group has a property $(S)$. [16] 


\subsubsection{Theorem. Conjugacy neighborhood theorem using convergence}

techniques. Let $X$ be a $G$-space where $G$ is Hausdorff locally compact with property $(\boldsymbol{S})$ and let $x \notin J(x)$ for every $x \in X$. Then for a point $x \in X$, there is a neighborhood $U$ of $x$ in $X$ such that the stabilizers of points in $U$ are conjugate to a subgroup of $G_{x}$.

Proof. Suppose that there is a point $x \in X$ such that for every neighborhood $U$ of $x$ there is $y \in U$ such that $G_{y} \not \subset G_{x}$. We can choose the neighborhoods system $U_{\alpha}$ of the point $x$ in $X$. We can find for every $\alpha$ there is a point $x_{\alpha} \in U_{\alpha}$ and $g_{\alpha} \in$ $G_{x_{\alpha}}$ such that $g_{\alpha} \notin G_{x}$. We have $g_{\alpha} x_{\alpha}=x_{\alpha}$ but $g_{\alpha} x \neq x$. By our choice of the neighborhoods of $x$, we have $x_{\alpha} \rightarrow x$ and $g_{\alpha} x_{\alpha}=x_{\alpha} \rightarrow x . x \notin J(x)$ implies that $g_{\alpha} \rightarrow g$ for some $g \in G$. By the continuity of the action, we have $g_{\alpha} x_{\alpha} \rightarrow g x$ and $g x=x$. Thus, $g \in G_{x}$. Now $g_{\alpha} \rightarrow g$ so that $k_{\alpha}=g^{-1} g_{\alpha} \rightarrow e$ where $e$ is the identity element in $G$. Let $V$ be a symmetric neighborhood of $e$ in $G$ with $\bar{V}$ is compact. Since $G$ has the property $(\boldsymbol{S})$, we can construct a sequence of neighborhoods $\left\{U_{n}\right\}$ of $e$ with $U_{n} \supseteq U_{n+1}$ and $\cap U_{n}=\{e\}$. On other the hand, since $k_{\alpha} \rightarrow e$, it allows us to construct a sequence $y_{n}=k_{\alpha_{n}} \neq e$ with $y_{n} \in U_{n}$ for every $n$. Let $W$ be a neighborhood of $e$ such that $W^{2} \subset V$. By our assumption, for every $n$ there is an integer $m_{n}$ such that $y_{n}^{2^{m_{n}}} \notin W$ so we can consider $m_{n}$ being the smallest integer satisfies this property for every $n$. Now, the inequality $2^{m_{n}-1}<2^{m_{n}}$ implies $y_{n}^{2^{m_{n-1}}} \in W$ and $y_{n}^{2^{m_{n-1}}} y_{n}^{2^{m_{n-1}}}=y_{n}^{2^{m_{n}}} \in W^{2} \subset V$. Since the closure of $V$ is compact then there is a subsequence $y_{n^{\prime}}$ of $y_{n}$ such that $y_{n^{\prime}}=$ $y_{n^{\prime}}^{2^{m^{\prime}}} \rightarrow a$ in $\bar{V}$. The condition $y_{n}^{2^{m_{n}}} \notin W$ implies that $a \notin W$ and $a \neq e$. Let $K$ be the set of the existence limits of such subsequences. We claim that $K$ is subgroup of $G$. Pick $a, b \in K$, then $a=\operatorname{limit}_{n^{\prime}}^{2^{m_{n^{\prime}}}}$ and $b=$ limity $2_{n^{\prime \prime}}^{2_{n^{\prime \prime}}}$. Put $s_{n}=2^{m_{n^{\prime \prime}}}-$ $2^{m_{n^{\prime}}}\left(\bmod 2^{m_{n^{\prime}}}\right)$. Then we have $s_{n} \leq 2^{m_{n^{\prime}}}$ and $\operatorname{limity}_{n^{\prime}}^{s_{n}}=e=b a^{-1} \in \bar{V}$. We have $b a^{-1} \in K$ and then $K$ is a subgroup of $G$ containing in $\bar{V}$ which contradicts 
that $G$ has property $(\boldsymbol{S})$. Therefore, there is a neighborhood of $x$ such that points in it whose stabilizers are subgroups of $G_{x} \mathbf{m}$. 


\section{Chapter Three}

\section{Weaker Forms of Proper Actions}

\subsection{Three Kinds of Proper Actions}

In the chapter two, we have stated two kinds of proper actions, Palais, and Bourbaki proper. We have mentioned that Palais proper implies Bourbaki proper. In general, the two actions are not equivalent. On the other hand, they have been put in different settings. There is another action that has been presented by Baum, Connes and Higson (the stronger one). The latter has been put in a setting different than Palais'. Biller in [8] has defined three weaker kinds of proper that are increasingly strong. In [8], the author makes three kinds of proper actions in unambiguous directions. There the author also establishes a weaker form termed Cartan depending on some of the properties of Palais' Cartan. In our view, the convergence technique makes the relation between three kinds of proper clearer and technique of proofs shorter and more understandable. Moreover, we shall show that our weaker forms are weaker than Biller's. In this way, we propose a new insight into the study of wider classes of proper action that use only convergence techniques. Before taking up those forms, we give Baum-Connes-Higson proper definition.

3.1.1 Definition. [6] Let $G$ be a locally compact and second countable $\left(T_{2}\right.$ included) and let $G$ be act continuously on a metrizable space $X$. The action of $X$ is called proper if for every $x \in X$ there is a triple $(U, H, \rho)$ such that:

1. $U$ is an open neighborhood of $x$, with $g y \in U$ for every $(g, y) \in G \times U$,

2. $H$ is a compact subgroup of $G$,

3. $\rho: U \rightarrow G / H$ is a $G$-map from $U$ to the homogeneous space $G / H$. 
3.1.2 Lemma. Let $G$ be a topological group and $g$ is an element of $G$ and $\mathcal{N}$ be a fundamental open neighborhoods system of the identity. Then for every $V \in \mathcal{N}$, there is an open neighborhood $U$ of $g$ such that $((U, U))=\{g \in G: g U \cap U \neq$ $\emptyset\} \subseteq V$

Proof. From topological group properties, especially for a fundamental open neighborhood system $\mathcal{N}$ of the identity element in $G$, we have that every element $V \in \mathcal{N}$, and any element $g \in G$ there is an element $N \in \mathcal{N}$ such that $N \subseteq$ $g V g^{-1}$. Then we have $((N, N)) \subseteq g V g^{-1}$ or $g^{-1}((N, N)) g \subseteq V$, and hence $((g N, g N)) \subseteq V$. Take $U=g N$.

3.1.3 Definition. [3] Let $X$ be a $G$-space. A subset $S$ of $X$ is called star if for every $x \in X$ there is a $g \in G$ such that the set $g x \in S$ and if, additionally, $S$ is also small then it is called strong.

3.1.4 Remark. We have shown that the extended sets $J(x)$ and $J_{S}(x)$, from the sets $E_{f}$ and $S E_{f}$ respectively, are good keys as to whether an action is Bourbaki proper or not. It is useful in this stage that we give explanations that one may gain from the set $J(x)$. First, let us prove the following theorem that we have stated it in the chapter two.

3.1.5 Theorem. Let $X$ be a $T_{2}$ space and let $G$ be a topological group acting continuously on $X$. The action is Bourbaki proper if and only if the set $J(x)=$ $\emptyset$ for every $x \in X$.

Proof. If the $G$ acts properly on $X$, suppose that there are points $y, x \in X$ and $y \in$ $J(x)$. There is a net $g_{\alpha} \rightarrow \infty$ in $G$ and a net $x_{\alpha} \rightarrow x$ in $X$ such that $g_{\alpha} x_{\alpha} \rightarrow y$. That is $\left(x_{\alpha}, g_{\alpha} x_{\alpha}\right) \rightarrow(x, y)$. Since $G$ acts properly on $X$, then there is a point $(g, x) \in$ $G \times X$ such that $\left(g_{\alpha}, x_{\alpha}\right) \rightarrow(g, x)$. This implies that $g_{\alpha} \rightarrow g$ which is a 
contradiction. Therefore, since $x, y$ are arbitrary, $J(x)=\emptyset$ for every $x \in X$. Conversely, to show that the condition $J(x)=\emptyset$ for every $x \in X$ implies that the map $\theta:(g, x) \rightarrow(x, g x)$ is proper, take a point $(x, y) \in X \times X$, then if $y \neq$ $g x$ for every $g \in G$, then $\theta^{-1}(x, y)=\emptyset$ and hence it is quasi compact. Suppose that $y=g x$ for some $g$ in $G$ and $\theta^{-1}(x, y)$ is not quasi compact. There is a net $\left(g_{\alpha}, x_{\alpha}\right) \rightarrow \infty$ in $\theta^{-1}(x, y)$ and hence in $G \times X$ since $\theta^{-1}(x, y)$ is closed subset of $G \times X$. Since $\theta\left(g_{\alpha}, x_{\alpha}\right)=\left(x_{\alpha}, g_{\alpha} x_{\alpha}\right)=(x, y) \rightarrow(x, y)$, then we have $x_{\alpha} \rightarrow$ $x$ and $g_{\alpha} x_{\alpha} \rightarrow y .\left(g_{\alpha}, x_{\alpha}\right) \rightarrow \infty$ implies that $g_{\alpha} \rightarrow \infty$ and $y \in J(x)$ which is a contradiction. Take $F$ as a closed subset of $G \times X$ and $(x, y) \in \overline{\theta(F)}$. There is a net $\left(g_{\alpha}, x_{\alpha}\right) \in F$ and $\theta\left(g_{\alpha}, x_{\alpha}\right)=\left(x_{\alpha}, g_{\alpha} x_{\alpha}\right) \rightarrow(x, y)$. So $x_{\alpha} \rightarrow x$ and $g_{\alpha} x_{\alpha} \rightarrow y$. Since $J(x)=\emptyset$, the net $g_{\alpha}$ must have a convergent point in $G$, say $g$. That is $g_{\alpha} \rightarrow$ $g$ and $x_{\alpha} \rightarrow x$ or $\left(g_{\alpha}, x_{\alpha}\right) \rightarrow(g, x) \in F$, since $F$ is closed. The continuity of $\theta$ implies to $\theta\left(g_{\alpha}, x_{\alpha}\right)=\left(x_{\alpha}, g_{\alpha} x_{\alpha}\right) \rightarrow \theta(g, x)=(x, g x)=(x, y)$ by $T_{2}$ condition of $X$. Therefore $\theta(F)$ is closed in $X \times X$ and $\theta$ is closed map.

3.1.6 Remark. If $X$ is not $T_{2}$ in the theorem (3.1.4), then the second direction may not be true. For example, $G=\{ \pm 1\}$ with the discrete topology, and $X=\mathbb{R}^{2}$ with the indiscrete topology. Then $G$ acts continuously on $X$ by $t(x, y)=$ $(x, t y)$ for every $t \in G$ and $(x, y) \in X$. Obviously, then $J((x, y))=$ $\emptyset$ for every $(x, y) \in X$. However, $X / G$ is not $T_{2}$ (not Bourbaki proper).

3.1.7 Proposition. [27] Let $X$ be a Cartan $G$-space (in the sense of Palais) and $x$ be a point in $X$. If $U$ is a neighborhood of $G_{x}$, then there is a neighborhood $V$ of $x$ such that $((V, V)) \subseteq U$. 


\subsection{Biller's Cartan Action and Weaker Cartan Actions}

3.2.1 Definition. [8] Let $X$ be a topological space and $G$ be a topological group. An action of $G$ on $X$ is said to be Cartan if the following two conditions are satisfied:

1. All stabilizers are quasi compact.

2. If $x \in X$, and $V_{G_{x}}$ is a nieghborhood of $G_{x}$, there is a neighborhood $U_{x}$ of $x$ such that $\left(\left(U_{x}, U_{x}\right)\right) \subseteq V_{G_{x}}$.

3.2.2 Proposition. Cartan action in the sense of Biller implies to the condition that $x \notin J(x)$ for every $x \in X$.

Proof. Assume that the two conditions of Biller be satisfied. Suppose that there is a point $x \in X$ such that $x \in J(x)$. Then these a net $g_{\alpha} \rightarrow \infty$ in $G$ and a net $x_{\alpha} \rightarrow x$ in $X$ such that $g_{\alpha} x_{\alpha} \rightarrow x$. For each $g \in G_{x}$ there is, since $g_{\alpha} \rightarrow \infty, \delta_{g} \in$ $D$ and $V_{g}$ is a nieghborhood of $g$ such that $g_{\alpha} \notin V_{g}$ for every $\alpha \geq \delta_{g}$. The collection $\left\{V_{g}\right\}$ forms an open cover of $G_{x}$ which is a quasi-compact, then there is a finite sub-collection $\left\{V_{g_{i}}\right\}_{i=1}^{n}$ covering $G_{x}$. Then we find $\delta \in D$ such that $g_{\alpha} \notin V_{g_{i}}$ for every $\alpha \geq \delta$, and $i \in\{1,2,3, \ldots, n\}$. Put $V=\cup_{i=1}^{n} V_{g_{i}}$ then $V$ is a neighborhood of $G_{x}$ and $g_{\alpha} \notin V$ for every $\alpha \geq \delta$. By the second condition of Biller, these is a neighborhood $U_{x}$ such that $\left(\left(U_{x}, U_{x}\right)\right) \subseteq V$. Since $x_{\alpha} \rightarrow x$ and $g_{\alpha} x_{\alpha} \rightarrow x$, then there is $\beta \in D$ such that $x_{\alpha} \in U_{x}$ and $g_{\alpha} x_{\alpha} \in U_{x}$ or $g_{\alpha} \in\left(\left(U_{x}, U_{x}\right)\right)$ for every $\alpha \geq \beta$. Choose $\gamma \geq \beta$ and $\gamma \geq \delta$, then we have $g_{\alpha} \in\left(\left(U_{x}, U_{x}\right)\right) \subseteq V$ and $g_{\alpha} \notin V$ for every $\alpha \geq \gamma$ which a contradiction, $x$ is an arbitrary point in $X$ and hence $x \notin J(x)$ for every $x \in X$. 


\subsubsection{Remark.}

- The converse of the above proposition is not true in general. For example, consider the action $t(x, y)=(x, t y)$ by the discrete topological group $G=$ $\{+1,-1\}$ acting on the plane with the indiscrete topology. Take a point $x=$ $(2,3)$ in the plane, then $G_{x}=\{1\}$. The subset $V=\{1\}$ of $G$ is an open neighborhood of $G_{x}$. However, there is a unique neighborhood $U=\mathbb{R}^{2}$ of $x$ and $((U, U))=\{-1,1\} \not \subset V$. It is obvious that $x \notin J(x)$.

- Under the second condition of definition of Biller Cartan action, the first condition is equivalent to the condition $x \notin \Lambda(x)$ for every $x \in X$.

In this stage, we have shown that there is a convergence condition that is weaker than Biller conditions. A fortiori one could define a weaker form of Cartan action by using such convergence techniques. We will call our version weak Cartan or just $w$-Cartan.

3.2.4 Definition. (New version of Cartan action) Let $X$ be topological space and $G$ be a topological group acts continuously on $X$. The action of $G$ on $X$ is said to be $w$-Cartan if the following condition is satisfied: $\forall x$ in $X, x \notin J(x)$.

3.2.5 Proposition. In the sense of Palais, Cartan and $w$-Cartan coincide [see, proposition (2.4.2)].

3.2.6 Proposition. Let $X$ be a $T_{2} w$-Cartan $G$-space and $x$ a point in $X$. Then the map $g \rightarrow g x$ is an open map from $G$ onto $G x$.

Proof. Let $V$ be an open neighborhood of e in $G$. To show $V x$ is an open in $G x$. Suppose it is not. There is a net $g_{\alpha}$ in $G$ with $g_{\alpha} x$ is not eventually in $V x$ but it converges to $x$. If $g_{\alpha} \rightarrow \infty$, then $x \in \Lambda(x) \subseteq J(x)$ which contradicts that $X$ is $w$ Cartan. So, $g_{\alpha} \rightarrow g$ for some $g \in G$. Then $g_{\alpha} x \rightarrow g x=x$ and hence $g \in G_{x}$. 
However, $g_{x} x \notin V x$ is equivalent to $g_{\alpha} \notin V G_{x}$ which is a neighborhood of $g$. This contradiction proves the proposition.

3.2.7 Proposition. Let $X$ be a $w$ - Cartan $G$-space and $x$ a point in $X$. Then the map $G / G_{x} \rightarrow G x$ is a homeomorphism.

Proof. It is obvious that the map is a bijection map, and it is also equivariant. Let $U$ be an open subset of $G / G_{x}$, then $\pi^{-1}(U)=U G_{x}$ is open in $G$ where $\pi$ is the canonical map from $G$ onto $G / G_{x}$. By the above lemma, $U x$ is open in $G x$.

3.2.8 Example. Let $G=\mathbb{Q}$ be the group of rational numbers with usual addition operation and the relative topology of the usual topology on $\mathbb{R}$, and let $G$ act on itself by the usual translations. That is, the action $(g, x) \rightarrow g+x$ for each $g, x \in$ $\mathbb{Q}$. Then $G$ acts continuously on itself. Also, the orbit space is just one point. Now for every net $g_{\alpha} \rightarrow \infty$ then either $g_{\alpha}$ goes to $\pm \infty$ or $g_{\alpha}$ converges to an irrational number $r$, and in the both cases if there is a net $x_{\alpha} \rightarrow x \in \mathbb{Q}$, then $g_{\alpha}+x_{\alpha} \rightarrow \infty$. So $x \notin J(x)$ for each $x \in \mathbb{Q}$. This action is not Cartan in the sense of Palais because $0 \in \mathbb{Q}$ and for any neighborhood $V$ of 0 , the set $((V, V))$ has not compact closure in $\mathbb{Q}$.

\subsubsection{Definition. (New versions of $\boldsymbol{w}$-proper actions) Let $X$ be a topological} space and $G$ be a topological group and let $G$ acts continuously on $X$. Then the action $G$ on $X$ is said to be:

i- $w$-proper action if is $w$-Cartan and the orbit space $X / G$ is Hausdorff.

ii- $w$-Palais proper if it is $w$-Cartan and the orbit space $X / G$ is regular $\left(T_{2}\right.$ is included).

iii- $w$-strongly proper if it is $w$-Cartan and the orbit space $X / G$ is paracompact ( $T_{2}$ is included). 
3.2.10 Remark. It is obvious that every $w$-strongly proper action is a $w$-Palais proper action and the latter is a $w$-proper action. A good universal example for converse sides is given by Biller in [8] as following; Let $G$ be a topological group and $X$ is a topological space. Let $G$ acts on the space $G \times X$ by $g(t, x)=(g t, x)$ for every $g, t \in G$ and $x \in X$. To show this action is $w$-Cartan. Take a point $\bar{z}=$ $(t, x) \in G \times X$. Suppose that $\bar{z} \in J(\bar{z})$, there is a net $\left(t_{\alpha}, x_{\alpha}\right) \rightarrow(t, x)$ in $G \times X$ and a net $g_{\alpha} \rightarrow \infty$ such that $g_{\alpha}\left(t_{\alpha}, x_{\alpha}\right)=\left(g_{\alpha} t_{\alpha}, x_{\alpha}\right) \rightarrow(t, x)$. Then we have that $g_{\alpha} t_{\alpha} \rightarrow t$ and $x_{\alpha} \rightarrow x$. By the previous lemma (3.1.2), if $V$ is a neighborhood of $e$, there is a neighborhood $U$ of $t$ such that $((U, U)) \subseteq V$. Since $g_{\alpha} \rightarrow \infty$, there is $\delta \in D$ such that $g_{\alpha} \notin V$ for every $\alpha \geq \delta$. Since $g_{\alpha} t_{\alpha} \rightarrow t$, and $t_{\alpha} \rightarrow t$ there is $\beta \in D$ such that $g_{\alpha} t_{\alpha} \in U$, and $t_{\alpha} \in U$ for every $\alpha \geq \beta$. So, for $\alpha \geq \delta$ and $\alpha \geq \beta$, we have $g_{\alpha} \in((U, U))$ and $g_{\alpha} \notin V$ is impossible. Therefore, $\bar{z} \notin J(\bar{z})$ for every point $\bar{z}=(t, x) \in G \times X$ and the action is $w$-Cartan. Moreover, the orbit space of this action is homeomorphic to $X$, as a result there is $w$-proper action which is not $w$-Palais proper and $w$-Palais proper which is not $w$-strongly proper.

3.2.11 Proposition. $w$-proper action is equivalent to the Bourbaki proper if $X$, in addition, is $T_{2}$.

Proof. Bourbaki proper implies that $J(x)=\varnothing$ for every $x \in X$. That is, $x \notin J(x)$ and hence $w$-Cartan. Also, the condition implies that for every pair of points $x, y \in$ $X$, we have $y \notin J(x)$ which implies to $X / G$ is $T_{2}$ whenever $X$ is $T_{2}$. Conversely, if $X$ is a $w$-proper, then $x \notin J(x)$ and since the orbit space is $T_{2}$. The condition $y \notin$ $J(x)$ for every pair of points $x, y \in X$ with $G x \neq G y$ implies that $J(x)=\varnothing$ for every point $x \in X$, and the latter implies that $X$ is Bourbaki proper whenever $X$ is $T_{2}$. 
3.2.12 Proposition. In the Palais setting, that is, $G$ is a locally compact $\left(T_{2}\right.$ included) and $X$ is a completely regular $\left(T_{3 \frac{1}{2}}\right)$, Palais proper and $w$-Palais proper coincide.

Proof. If $X$ is a $w$-Palais proper, then it is a $w$-Cartan which is under Palais setting $X$ is also Palais Cartan action from the previous proposition. Moreover, $X / G$ is regular by the definition of $w$-Palais proper. Thus, $X$ is Palais proper (since Cartan with regularity of the orbit space is equivalent to Palais proper). The proof of the other side is obvious.

3.2.13 Proposition. If $X$ is a $T_{2} G$-space, $A$ is an open thin subset of $X$, then $G A$ is $w$-proper (also Bourbaki) $G$-space $(\cong J(x)=\emptyset$, for every $x \in G A)$.

Proof. To show that the action $G \times G A \rightarrow G A$ is $w$-proper. It must be $w$-Cartan $G$ space and the orbit space $G A / G$ is Hausdorff. To show that for every point $x \in$ $G A, x \notin J(x)$. Suppose that is not true. That is, there is a point $g_{\mathrm{o}} x \in G A$, but $g_{\mathrm{o}} x \in$ $J\left(g_{0} x\right)$. Then $\exists x_{\alpha} \rightarrow g_{0} x$ in $G A$ an $d \exists g_{\alpha} \rightarrow \infty$ in $G$ such that $g_{\alpha} x_{\alpha} \rightarrow g_{0} x$ in $G A$. Since $x \in A$ and $g_{\circ}^{-1} g_{\alpha} x \rightarrow x$, then there is $\delta \in D$ such that $g_{\circ}^{-1} g_{\alpha} x \in A$ for every $\alpha \geq \delta$, so $g_{\circ}^{-1} g_{\alpha} \in((A, A))$ which has a quasi-compact closure. Then there is a $g \in G$ and $g_{\circ}^{-1} g_{\alpha} \rightarrow g$ or $g_{\alpha} \rightarrow g_{\circ} g$ which is a contradiction. Therefore, for every $x \in G A, x \notin J(x)$. To show that $G A / G$ is $T_{2}$. Suppose that there are $\bar{x}, \bar{y} \in$ $G A / G$ such that $\bar{x} \neq \bar{y}$ and $y \in J(x)$ where $x \in \bar{x}$ and $y \in \bar{y}$, then there are $g_{\circ}, g_{1} \in G$ such that $a_{\circ}=g_{\circ} x \in A$ and $a_{1}=g_{1} y \in A$. Since $y \in J(x)$, then $a_{1} \in$ $J\left(a_{\circ}\right)$, then there are a net $g_{\alpha} \rightarrow \infty$ and $x_{\alpha} \rightarrow a_{\circ}$ such that $g_{\alpha} x_{\alpha \rightarrow a_{1}}$. $A$ is open, then there is $\delta \in D$ such that $x_{\alpha} \in A$ and $g_{\alpha} x_{\alpha} \in A$ for each $\alpha \geq \delta$. This means that $g_{\alpha} \in \overline{((A, A))}$ which is quasi compact. So $g_{\alpha} \rightarrow g$ for some $g \in G$ which is a contradiction. Therefore, $G A / G$ is $T_{2}$, and the action is $w$-proper. 
3.2.14 Corollary. In the Palais setting, for a $w$-Cartan $G$-space $X$, every point has a neighborhood $N$ such that $G N$ is Palais proper.

Proof. It follows from the fact that Cartan and $w$-Cartan coincide in the Palais setting.

3.2.15 Remark. If $X$ is a $G$-space and $A$ is a closed thin subset of $X$, then $G A$ is not necessarily to be closed in $X$. For example, a subset

$A=\left\{(x, y) \in \mathbb{R}^{2}:(x-16)^{2}+y^{2} \leq 1\right\}$ is a closed thin in the $G$-space $\mathbb{R}^{2}$, where $G$ is the set of real numbers with the usual topology acts on $\mathbb{R}^{2}$ by the formula $t(x, y)=\left(x e^{-t}, y e^{t}\right)$. However, $G A$ is not closed. That is, $\pi(A)$ is not closed in the orbit space $X / G$. If $A$ is a small subset of a $T_{2} G$-space, then we can obtain the following results.

3.2.16 Proposition. Let $A$ be a small subset of a $T_{2} G$-space $X$, then $G$ acts on $G A$ $w$ - properly.

Proof. First, we show $J(x)=\emptyset$ for every $x \in G A$. Suppose there are points $x=g_{1} a_{1}, y=g_{2} a_{2} \in G A$ such that $y \in J(x)$. Then there is a net $g_{\alpha} a_{\alpha} \rightarrow g_{1} a_{1}$ in $G A$ and a net $t_{\alpha} \rightarrow \infty$ such that $t_{\alpha} g_{\alpha} a_{\alpha} \rightarrow g_{2} a_{2}$. Take $U$ be a neighborhood of $a_{1}$ in $X$ such that a set $((A, U))$ has a quasi-compact closure. Since $g_{1}^{-1} g_{\alpha} a_{\alpha} \rightarrow a_{1}$ and $a_{\alpha} \in A$, thus $g_{1}^{-1} g_{\alpha} \in((A, U))$ which implies to there is a $g \in G$ such that $g_{1}^{-1} g_{\alpha} \rightarrow g$ or $g_{\alpha} \rightarrow g_{1} g$. Similarly, there is a neighborhood $V$ of $a_{2}$ in $X$ such that $((A, V))$ has a quasi-compact closure. Then, we have $g_{2}^{-1} t_{\alpha} g_{\alpha} \in((A, V))$ which implies to there is a $t \in G$ such that $g_{2}^{-1} t_{\alpha} g_{\alpha} \rightarrow t$ or $t_{\alpha} \rightarrow g_{2} t g_{1} g$ which is a contradiction. Since $x, y$ are arbitrary points in $G A$, therefore, $J(x)=\emptyset$ for every $x \in G A$. It is obvious that $G A$ is a subspace of $T_{2}$ space. Therefore, $G A$ is a $T_{2}$ and $w$-proper $G$-space. 
3.2.17 Proposition. [4] If $A$ is a small subset of a $G$-space $X$, then $\bar{A}$ is also small. Moreover, if $f: X \rightarrow Y$ is a morphism ( $G$-map) of $G$-spaces $Y$ and $X$, then $f^{-1}(A)$ is a small subset of $Y$.

3.2.18 Proposition. Let $X$ be a $T_{2} G$-space and let $S$ be a strong subset of $X$, then $X$ is $w$-proper.

Proof. From the proposition (3.2.16), $G S=X$ is a $w$-proper.

3.2.19 Proposition. Let $X$ be a $T_{2} G$-space and $A$ be a closed small subset of $X$, then:

a) The restriction map $\rho$ of the action on $G \times A$ into $X$ is a proper map.

b) The restriction map $\varphi$ of the orbit map on $A$ into $X / G$ is a proper map.

Proof. For $(\boldsymbol{a})$, to show $\rho$ is a proper map, it suffices to show that its exceptional set $E_{\rho}$ is empty. Suppose that there is a point $x \in E_{\rho}$, then there is a net $\left(g_{\alpha}, a_{\alpha}\right) \rightarrow$ $\infty$ in $G \times A$ such that $g_{\alpha} a_{\alpha} \rightarrow x$. Since $A$ is small, there is a neighborhood $U_{x}$ of $x$ such that the set $\left(\left(A, U_{x}\right)\right)$ has quasi-compact closure. $g_{\alpha} a_{\alpha} \rightarrow x \in U_{x}$, then there is $\delta \in D$ such that $g_{\alpha} a_{\alpha} \in U_{x}$ for every $\alpha \geq \delta$ that implies, since $a_{\alpha} \in A$, to $g_{\alpha} \in$ $\left(\left(A, U_{x}\right)\right)$ which has quasi compact closure, so there is $g \in G$ such that $g_{\alpha} \rightarrow g$ and then $g_{\alpha}^{-1} \rightarrow g^{-1}$ and $g_{\alpha}^{-1}\left(g_{\alpha} a_{\alpha}\right)=a_{\alpha} \rightarrow g^{-1} x \in A$, since $A$ is closed. We have that $\left(g_{\alpha}, a_{\alpha}\right) \rightarrow\left(g, g^{-1} x\right)$ which is a contradiction and $E_{\rho}=\phi$. Since $X$ is $T_{2}$, then $\rho$ is proper. To prove $(\boldsymbol{b})$, first we show that $G A$ is closed in $X$. Take $x \in$ $\overline{G A}$. Then there is a net $g_{\alpha} a_{\alpha} \rightarrow x$ and $g_{\alpha} a_{\alpha} \in G A$. Since $A$ is small, there is a neighborhood $U$ of $x$ in $X$ such that the set $((U, A))$ has quasi-compact closure. Since $g_{\alpha} a_{\alpha} \in U$ for some $\alpha \geq \delta$ and $g_{\alpha}^{-1}\left(g_{\alpha} a_{\alpha}\right)=a_{\alpha}$. Thus, $g_{\alpha}^{-1} \in((U, A))$ and there is a $g \in G$ such that $g_{\alpha}^{-1} \rightarrow g$. Also, we get $a_{\alpha} \rightarrow g^{-1} x \in A$ since $A$ is closed. Therefore, $g\left(g^{-1} x\right)=x \in G A$ and $G A$ is closed in $X$. GA is $G$ - invariant 
and hence $G A / G$ is closed in $X / G$. Now, suppose that $S E_{\varphi} \neq \varnothing$, that is, there is a point $\tilde{x}$ in $S E_{\varphi}$. Thus, there is a net $a_{\alpha}$ in $A$ such that $a_{\alpha} \rightarrow \tilde{x}$ and $\operatorname{clu}(a) \cap$ $\varphi^{-1}(\tilde{x})=\emptyset$ in A. Since $\varphi^{-1}(\tilde{x})=((A,\{x\})) x$ which is closed since $A$ is closed and quasi-compact since $A$ is small. $\mathrm{X}$ is $T_{2}$, there is a neighborhood $U$ of $\varphi^{-1}(\tilde{x})$ which does not meet $\operatorname{clu}\left(a_{\alpha}\right)$ this implies to $a_{\alpha} \nrightarrow \tilde{x}$ and a contradiction. Therefore, $S E_{\varphi}=\emptyset$.

\section{$w$-Action by locally quasi-compact groups}

Our aim is to put the three kinds of proper actions in clear implications under suitable conditions. Palais setting is reasonable, so we shall not go away form those conditions. The following shows the analogous properties that are still true for our forms of $w$-actions and we need $G$ is a locally quasi-compact and $X$ is $T_{2}$.

3.2.20 Lemma. Let $G$ be a topological group. Then

a) For every quasi-compact subset of $G$ its closure is still a quasi-compact,

b) if $G$ is a locally quasi compact and $K$ is a subset of $G$, then $\bar{K}$ is a quasicompact if and only if $K$ contained in a quasi-compact closed neighborhood.

Proof. The proof for $(\boldsymbol{a})$, it is follows that if $K$ is a quasi-compact, then $\bar{K}=\overline{\{e\}} K$ [19] which is a quasi-compact, $e$ is the identity element in $G$. For $(\boldsymbol{b})$, it is obvious that if $K \subseteq V$, where $V$ is closed quasi-compact neighborhood of $K$, then $\bar{K} \subseteq V$, and then $\bar{K}$ is quasi-compact. Conversely, if $K$ has quasi-compact closure, then for every $g \in \bar{K}$ there is quasi-compact neighborhood $V_{g}$ and hence by $(\boldsymbol{a})$, there is a quasi-compact $\overline{V_{g}}$. So $\bar{K} \subseteq \bigcup_{g \in \bar{K}} \bar{V}_{g_{i}}$, then there is a finite set $g_{1}, \ldots, g_{n}$ such that $\bar{K} \subseteq \cup_{i=1}^{n} \bar{V}_{g_{i}}$. Choose $V=\cup_{i=1}^{n} \bar{V}_{g_{i}}$. Then $V$ is a quasi-compact closed neighborhood of $K$. 
3.2.21 Proposition. Let $G$ be a locally quasi- compact topological group acts continuously on $X$, and for every $x \in X$ the subspace $G x$ is $T_{2}$. Then the action of $G$ on $X$ is $w$-Cartan if and only if for every point $x \in X$ has a thin neighborhood.

Proof. Assume that the action is $w$-Cartan. Since $x \notin J(x)$, then by proposition. (2.4.6) there is a neighborhood $U$ of $x$ and a quasi-compact neighborhood $V$ of the identity such that $\overline{((U, U))} \subseteq \bar{V}$ which is a quasi-compact, that is $x$ has a thin neighborhood. The converse is obvious.

3.2.22 Example. Let $G=\{1,-1\}$ be a group with discrete topology and let $X$ be the plane with a topology structure is identified by open sets $\emptyset, X, U_{1}, U_{2}$ where $U_{1}$ is open upper half of the plane and $U_{2}$ is the open lower half of the plane. Then a map $G \times X \rightarrow X$ is given by the formula $\pm 1 .(x, y)=(x, \pm y)$ is an action. The continuity of the map come from that the inverse image of $U_{i}, i=1$ or 2 is the set $\left.\left\{\{1\} \times U_{i}\right\} \cup\{-1\} \times U_{j}\right\}$ which is open in $G \times X$, where $j=1$ if $i=2$ and vice versa. $X$ is not $T_{2}$ but every orbit is $T_{2}$ space of $X$. Also, every point has a thin neighborhood since $G$ is compact. $X$ is not Cartan $G$-space in the sense of Palais since $U_{1}$ is thin but $G U_{1}=X \backslash L$ is not Palais proper, where $L$ is the set of points on $x$-axis. By proposition (3.2.21), $X$ is $w$-Cartan.

3.2.23 Theorem. ( $w$-proper actions by locally quasi-compact groups) Let $G$ be a locally quasi-compact group acts continuously on a $T_{2}$ topological space $X$. Then the action is:

i- $\quad w$-proper if and only if every pair of points of $X$ has relatively compact neighborhoods.

ii- $w$-Palais proper if and only if $X$ is regular and each point has small neighborhood. 
iii- $W$-strongly proper if and only if $X$ is paracompact and there is an open strong subset of $X$.

Proof. For $(i)$, assume that the second side in $(i)$ is satisfied. Suppose that there a pair of points $x, y \in X$ such that $y \in J(x)$. Then there is a net $x_{\alpha} \rightarrow x$ in $X$ and a net $g_{\alpha} \rightarrow \infty$ in $G$ such that $g_{\alpha} x_{\alpha} \rightarrow y$. By the assumption, there are neighborhoods $U, V$ of $x, y$ respectively, such that $((U, V))$ has a quasi-compact closure. Also, since $x_{\alpha} \rightarrow x$ and $g_{\alpha} x_{\alpha} \rightarrow y$ there is $\delta \in D$ such that $x_{\alpha} \in U$ and $g_{\alpha} x_{\alpha} \in V$ or $g_{\alpha} \in((U, V))$ for every $\alpha \geq \delta$. The set $((U, V))$ has a quasi-compact closure, so there is $g \in G$ and $g_{\alpha} \rightarrow g$ which is a contradiction. Thus, $J(x)=\phi$ for every $x \in$ $X$. The last result with our assumption that $X$ is $T_{2}$ implies to that the action is Bourbaki proper. By proposition (3.2.11), $X$ is $w$-proper. Conversely, assume the action is $w$-proper and again by proposition (3.2.11), that means $J(x)=\emptyset$ for each $x \in X$. Suppose that there is a pair of points $x, y \in X$ such that every pair of neighborhoods $U_{x}$ and $U_{y}$ of $x, y$ respectively, the set $\left(\left(U_{x}, U_{y}\right)\right)$ has no a quasicompact closure. Since $J(x)=\varnothing$, then the set $((\{x\},\{y\}))$ is a quasi-compact. Let $V$ be a quasi-compact neighborhood of the set $((\{x\},\{y\}))$ and then $\bar{V}$ is still quasicompact by lemma (3.2.20). Then, by our hypothesis, $\left(\left(U_{x}, U_{y}\right)\right) \nsubseteq \bar{V}$ for every pair of neighborhoods of $x$, and $y$. We can construct a net $x_{\alpha} \rightarrow x$ in $X$ and a net $g_{\alpha}$ in $G$ such that $g_{\alpha} x_{\alpha} \rightarrow y$ and $g_{\alpha} \notin \bar{V}$. Since $J(x)=\emptyset$, then $g_{\alpha} \rightarrow$ $g$ for some $g \in G$. By the continuity of the action and $X$ is $T_{2}$, then we have $g_{\alpha} x_{\alpha} \rightarrow g x=y$ and $g \in((\{x\},\{y\})) \subseteq V$ and hence $g_{\alpha} \in V \subseteq \bar{V}$ which is a contradiction. Therefore, the statement is true. For $(i i)$, assume that the action is $w$-Palais proper. To show that every point in $X$ has small neighborhood, take $x \in X$. Since $J(x)=\emptyset, x$ has thin neighborhood, say $U_{x}$, and then $G U_{x}$ is a neighborhood of $G x$. By regularity of the orbit space, there is an invariant closed neighborhood $W$ of $G x$ in $X / G$ such that $x \in W \subseteq G U_{x}$. Put $S=U_{x} \cap W$. We 
claim that the subset $V$ is a small neighborhood of $x$ in $X$. If $y$ is an arbitrary point in $X$, then either $y \in G U_{x}$ or $y \in X \backslash G U_{x}$. If $y \in G U_{x}$, say $y \in g U_{x}$ for some $g \in$ $G$, then $g U_{x}$ is a neighborhood of $y$ and $\left(\left(g U_{x}, S\right)\right) \subseteq\left(\left(g U_{x}, U_{x}\right)\right)$ where the latter has a quasi-compact closure. If $y \in X \backslash G U_{x}$, then $X \backslash W$ is a neighborhood of $y$ and $((X \backslash W, S))=\varnothing$. So, in either case $y$ has a neighborhood which is a relatively thin to $S$. Thus $S$ is a small neighborhood of $x$. Since $x$ is an arbitrary point in $X$, every point in $X$ has small neighborhood. Now, pick a point $x \in X$ and let $S$ be a small neighborhood of $x$. Then the closure $\bar{S}$ is also small neighborhood of $X$. By proposition $(3.2 .19, b)$, the map $\bar{S} \rightarrow X / G$ is proper, so $\bar{S}$ is regular since the inverse image of regular space is regular under proper map and the domain space is $T_{2}$. We have that every point in $X$ has a closed neighborhood which is regular subspace of $X$. Therefore, $X$ is regular [11, I, prop.13]. Conversely, if every point has a small neighborhood then $J(x)=\varnothing$ for every $x \in X$ and hence every point has a small closed neighborhood $K$ which is regular and admits a proper map, by proposition $(3.2 .19, b)$, onto the orbit space $X / G$. Therefore, $X / G$ is regular. For ( $i i i)$, the proof of the first part, we adapt it from Abel [4, prop.1.8]. Since $X$ is $w$-Palais proper, there is a closed small subset $S$ of $X$ from part $i i$. Now for every $x \in X$ there is an open neighborhood $U_{x}$ such that $A \cap G U_{x} \subseteq U_{x}$. The open cover $\pi\left(U_{x}\right), x \in X$ has a locally finite open refinement $\left\{V_{x_{\alpha}}\right\}$ such that $V_{x_{\alpha}} \subseteq \pi\left(U_{x_{\alpha}}\right)$. Set $W_{\alpha}=U_{x_{\alpha}} \cap \pi^{-1}\left(V_{x_{\alpha}}\right)$. Then the family $\left\{W_{\alpha}\right\}$ is a locally finite of small subsets of $X$. Then $F=\cup W_{\alpha}$ is an open small star subset of $X$. Moreover, the product $G \times F$ is a quasi-regular and a quasi-paracompact. By proposition $(3.2 .19, a)$, the map $G \times F \rightarrow X$ is surjective proper, then $X$ is a quasi-paracompact. $X$ is $T_{2}$ and hence $X$ is also paracompact. For the other side, assume that $X$ is paracompact and $F$ is an open strong subset of $X$, then a map $\bar{F} \rightarrow X / G$ is proper, proposition $(3.2 .19, b)$. Since $\bar{F}$ is paracompact, then $X / G$ is also paracompact. Being there is a 
strong subset of $X$ implies to every point in $X$ has a thin neighborhood proposition (3.2.19), and hence $x \notin J(x)$. Therefore, the action is strongly proper.

The following facts in [4] then become results following from theorem (3.2.23). We sum them up in the following corollary.

3.2.24 Corollary. Let $G$ be a locally quasi-compact group acts continuously on $T_{2}$ space $X$ and the action of $G$ is $w$-Cartan proper. Then

a) If $X / G$ is a paracompact, $X$ has a strong set.

b) $X$ has a paracompact (closed) strong set if and only if $X / G$ is paracompact.

c) If $X / G$ is paracompact, then $X$ is paracompact.

3.2.25 Proposition. Let $G$ be a locally compact acting $w$-strongly properly on a metrizable space $X$. Then then $X / G$ is metrizable.

Proof. It follows from proposition (3.2.19) and theorem $(3.2 .23, \mathrm{iii})$ that there is a strong (also closed) subset $S$ of $X$ and there is a surjective proper mapping from $S$ onto the orbit space $X / G$. Metrizability is a proper map invariant.

\subsection{Application}

In this section, we show an application for limit sets, especially the weaker forms of $w$-actions in another topic that are related to the theory of $G$-spaces. It is well-known that if $X$ is a topological group and $G$ is a subgroup of $X$, there is a natural action of $G$ on $X$ is given by $g * x=x * g^{-1}$ for every $g \in G$, and $x \in X$. In [5], the author has answered the following open problem: 
Let $G$ be a locally compact group. Then the orbit space $X / G$ of any paracompact proper $G$-space $X$ is paracompact.

The author in that work has solved the above problem in a specific case, when a space $G$ is a topological subgroup of $X$. We shall show how our technique could be a good way to describe the above problem. Before we take up the main proof of the open problem, the following results can be established for an arbitrary case.

3.3.1 Proposition. Let $G$ be a topological subgroup of a group $X$, and let $G$ act on $X$ naturally. For $x \in X, x \notin J(x)$.

Proof. Suppose there is a point $x \in X$ and $x \in J(x)$. Then there are nets $g_{\alpha} \rightarrow$ $\infty$ in $G$ and $x_{\alpha} \rightarrow x$ in $X$ such that $g_{\alpha} * x_{\alpha}=x_{\alpha} \cdot g_{\alpha}{ }^{-1} \rightarrow x$ in $X$. By continuity of multiplication law of the group $X$, then $x_{\alpha}{ }^{-1} \rightarrow x^{-1}$ in $X$ and $g_{\alpha}{ }^{-1}=$ $x_{\alpha}^{-1}\left(x_{\alpha} \cdot g_{\alpha}{ }^{-1}\right) \rightarrow x^{-1} \cdot x=e$ in $X$ or $g_{\alpha} \rightarrow e$ in $X$ and then in $G$ which is a contradiction. Since the point $x$ is an arbitrary point in $G$, we have $x \notin J(x)$ for every point $x \in X$.

3.3.2 Remark. One can deduce that every natural action is $w$-Cartan.

3.3.3 Proposition. The natural action of $G$ on $X$ is $w$-Palais proper if and only if $G$ is closed subgroup of $X$.

Proof. If the action is $w$-Palais proper, then the orbit space $X / G$ is $T_{2}$ and this equivalent to $G$ is closed. Conversely, if $G$ is a closed subgroup of $X$, then $X / G$ is $T_{2}$ and hence it is also regular. By the proposition (3.3.1), we have $x \notin J(x)$ for every point $x \in X$. Therefore, the action is $w$-Palais proper.

3.3.4 Remark. One can deduce that the natural action is also $w$-proper when $G$ is closed subgroup of $X$. 
3.3.5 Proposition. Let $X$ be a Hausdorff topological group and $G$ be a closed subgroup of $X$, the natural action of $G$ on $X$ is Bourbaki proper.

Proof. This follows from remark (3.3.4) and proposition (3.2.11).

3.3.6 Proposition. Let $H$ be a subgroup of $G$. Then the natural action, $t(g H)=$ $\operatorname{tg}(H)$, of $G$ on $G / H$ is a $w$-Cartan if and only if $H$ is a quasi-compact subgroup of $G$.

Proof. Since the action of $G$ on $G / H$ is transitive, then $\Lambda(x)=J(x)$ for every $x \in$ $G / H$. Thus, $H \notin \Lambda(H)$ implies that the stabilizer subgroup of $H \in G / H$ under $G$, that is $G_{H}=\{g \in G: g H=H\}=H$ is quasi-compact. Conversely, if $H$ is quasicompact, suppose that the action is not $w$-Cartan. Then there is a point $g H$ in $G / H$ such that $g H \notin J(g H)$, then by proposition (2.4.6) there is a neighborhood $U_{g H}$ of $g H$ in $G / H$ and a quasi-compact neighborhood $V$ of the identity element in $G$ such that $\left(\left(U_{g H}, U_{g H}\right)\right) \subseteq V$. Then $g H \in J(g H)$ implies that there is a net $g_{\alpha} \rightarrow \infty$ in $G$ and a net $t_{\alpha} H \rightarrow g H$ in $G / H$ such that $g_{\alpha} t_{\alpha} H \rightarrow g H$. Thus, $g_{\alpha} \in\left(\left(U_{g H}, U_{g H}\right)\right) \subseteq$ $V$ which means the net $g_{\alpha}$ must have convergent subnet which is a contradiction.

\subsubsection{Remark.}

1. If $X$ is a Hausdorff topological group and $G$ is a locally compact subgroup of $X$, then the natural action of $G$ on $X$ has Palais setting because $X$ is completely regular ( $T_{2}$ included) and $G$ is a locally compact group.

2. Corollary (3.3.8) provides an argument for theorem (1.1) in [5].

3.3.8 Corollary. If $X$ is a $T_{2}$ topological group and $G$ is a locally compact subgroup of $X$, the natural action of $G$ on $X$ is Palais proper.

Proof. It follows from remark $(3.3 .7,1)$, proposition (3.3.3), and proposition (3.2.12). 
Now let us give a sketch for the proof of the suggested open problem using our notions. Under assumptions of theorem $(3.2 .23$, iii), we have that if $X$ is paracompact and it has an open strong subset, then it is equivalent to the action being $w$-strongly proper. That is, $X$ is $w$-Cartan and the orbit space is paracompact. Therefore, under the open problem assumption, the orbit space is paracompact if and only if $X$ has closed strong subset. The last claim always holds for a locally compact subgroup $G$ of $T_{2}$ group $X$ acting naturally on $X[\mathbf{5}, \mathbf{T h} ., \mathbf{1 . 2}]$. We can summarize the above discussion by the following proposition.

3.3.9 Proposition. Let $G$ be a locally compact subgroup of $T_{2}$ group $X$, and let $G$ act naturally on $X$. Then $X$ is paracompact if and only if the action is $w$-strongly proper.

Proof. It follows from theorem (3.2.23, $i i i)$ and the above proof sketch.

Finally, it is also interesting to refer in this stage to the advantage of the existence of a closed strong subset $A$ of $G$-space $X$. Proposition $(3.2 .19, b)$ admits a surjective proper map $A \rightarrow X / G$. Moreover, it is well-known that proper mapping and its inverse could be preserve many topological properties such as, for example, compactness, para-compactness, metrizability, and so on. In the following, we state more general result that it holds whenever a $G$-space has a strong subset.

3.3.10 Proposition. Let $P$ be a topological property preserved under proper maps and inherited by closed subsets. Assume that $X$ is a topological group with the property $P$ and let $G$ be a locally compact subgroup of $X$. Then the orbit space $X / G$ of the natural action also has the property $P$.

Proof. It follows from the fact that the natural action has closed strong set and proposition $(3.2 .19, b)$. 


\section{Chapter Four}

\section{Convergence Techniques for Slices}

The existence of slices in $G$-spaces has the advantage that it supports additional theorems in transformation group action theory. For example, the Conjugacy Theorem due to Montgomery, the Equivariant Embedding Theorem [27, 28, 29], and some other notions. In this Chapter, we discuss a slice theorem in the theory of $G$-spaces, especially the enough slice notion that has been presented by Biller in [8]. The convergence technique is used to verify the notion of enough slice with weaker forms of $w$-actions. We show that if $G$ is an arbitrary locally compact group acting on a completely regular $G$-space $X$, then the existence of enough slice at each point is equivalent to $X$ being $w$-Cartan. Moreover, Biller in [9] has defined an $H$-slice where $H$ is a closed subgroup of an arbitrary locally compact group acting on a completely regular space. Also, the author gives a characterization for the existence $H$-slice without assumption of Lie group on $G$. In the first section, we give helpful motivation for slice in an arbitrary $G$-space.

\section{Motivation for slices}

Let $G$ be a topological group and $H$ be a subgroup of $G$ and $A$ be a $H$-space. Then a twisted product of a fiber $A$ is the orbit space $G \times_{H} A$ of an $H$-action $\theta$ on the space $G \times A$ given by $h(g, a)=\left(g h^{-1}, h a\right)$. The $H$-orbits are denoted by $H(g, a)$ or $[g, a]$, and the canonical quotient map is denoted by $q: G \times A \rightarrow$ 
$G \times_{H} A$ is given by $(g, a) \mapsto[g, a]$. It is clear that $q$ is an open map. The orbit space may be considered as a $G$-space and takes the form $g\left[g_{1}, a\right]=\left[g g_{1}, a\right]$. Then $q$ may be considered as $H$-map. Take $S=q(e, A)$. Then $S$ satisfies the following natural three properties [21].

1. $((S, S))=H$.

2. $S$ is an $H$ - invariant.

3. The map $G \times S \rightarrow G \times_{H} A$, given by $(g, s) \rightarrow g s$, is open.

Let us now call $S$ an $H$-slice in $G \times_{H} A$. On the other hand, a natural question may arise concerning the conditions under which an arbitrary $G$-space $X$ can be written as $G \times{ }_{H} A$ where $A$ is an arbitrary $H$-space. In this case, one may construct, in a natural way, an $H$-slice $S$ in $X$. Let $X$ be a $G$-space and $A$ is a subset of $X$ such that $((A, A)) A=A$, then $((A, A))$ is a subgroup of $G$, say $H$. Also, we can easy construct the twisted product $G \times{ }_{H} A$ with fiber $A$ and a quotient map $q: G \times A \rightarrow$ $G \times{ }_{H} A$. Also, one can consider a map $G \times A \rightarrow X$ is given by $(g, a) \mapsto g a$ which is a restriction of the $G$-action on $G \times A$ and it is always considered as an $H$-map. Moreover, we can construct a map from the two previous maps, say $\tau: G \times_{H} A \rightarrow X$ given as $[g, a] \rightarrow g a$, which is always injection, that makes the following diagram is commutative.

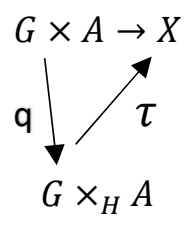

According to our aim, if the map $G \times A \rightarrow X$ is an open (or closed), then the map $\tau$ is an embedding onto an open subspace of $X$. Since the diagram commutes, we 
have that $G \times_{H} A \cong G A \subseteq X$. In this case the subset $S=\tau(q(e, A)) \subseteq G A$ is an $H$ slice in $G A$ which is open in $X$, and so $S$ is also an $H$-slice in $X$. On the other hand, suppose there is a $G$-map $f: X \rightarrow G / H$ of $G$-spaces $X$ and $G / H$. Take $S=f^{-1}(H)$. Then $S$ is an $H$-space and the twisted product $G \times{ }_{H} S$ with fiber $S$ exists. By the same procedure, and because the map $\tau$, in this case, is even onto (since $f$ is a $G$ map), then it is a homeomorphism onto $X$ whenever the map $G \times S \rightarrow X$ is open or closed and so we have $S$ is an $H$-slice in $X$ and $X \cong G S \cong G \times_{H} S$.

\subsection{Slices}

There are several definitions for the slice notion in the $G$-space theory, and here we recall the most well-known ones. In general, they are not equivalent, but if $G$ is a locally compact group acting on $T_{2}$ space, then they coincide.

4.1.1 Definition. (Slice in Palais Sense). Let $G$ be a locally compact group acting on a completely regular space $X$ and let $H$ be a closed subgroup of $G$. A subset $S$ of $X$ is said to be an $H$-slice provided that $G S$ is open in $X$ and there is $G$-map $f: G S \rightarrow G / H$ such that $f^{-1}(H)=S$. By a slice at $x$ we mean a $G_{x}$-slice in $X$ which contains $x$.

4.1.2 Proposition. [29] Let $X$ be a $G$-space and let $H$ be a closed subgroup of $G$. Then the following are equivalent:

a) $H$ is compact and $S$ is an $H$-slice in $X$.

b) $S$ has the following properties:

1. $S$ is closed in $G S$, and $G S$ open in $X$,

2. $S$ is $H$-invariant,

3. $((S, S))=H$, 
4. $S$ has a thin neighborhood in $G S$.

4.1.3 Theorem. (Palais Slice Theorem). Let $G$ be a Lie group. If $X$ is a Cartan $G$-space (in the sense of Palais), then there exists a slice at each $x$ in $X$.

4.1.4 Lemma. [9] Let $G$ be a locally compact group acting on a Hausdorff space $X$ and let $H$ be a closed subgroup of $G$. We call a nonempty subset $S \subseteq X$ an $H$-slice in $X$ for the action of $G$ if any one of the following equivalent conditions is satisfied:

1. There is a $G$-invariant open subset $U \subseteq X$ that admits a $G$-equivariant map $\varphi: U \rightarrow G / H$ such that $S=\varphi^{-1}(H)$. (Note that $U=G S$, and $\varphi$ is determined uniquely by $S$ because $\varphi(g s)=g \varphi(s))$.

2. There is an $H$-space $A$ and a $G$-equivariant open embedding $\psi: G \times_{H} A \rightarrow X$ such that $\psi([e, A])=S$.

3. The subset $S$ is invariant under $H$, the subset $G S \subseteq X$ is open, and $\chi: G \times_{H} S \rightarrow G S$ is given by $[g, s] \rightarrow g s$ is a homeomorphism.

Before taking up the enough slice notion [8], we shall review some natural properties of twisted product.

4.1.5 Proposition. The action $H$ on $G \times A$ is free and the map $\theta$ is proper whenever $H$ is quasi-compact.

Proof. Take $h \in H$, and $(g, a) \in G \times A$ and let $h(g, a)=(g, a)$. Then $\left(g h^{-1}, h a\right)=(g, a)$ which implies to $g h^{-1}=g$ and hence $h=e$. Assume $H$ is quasi-compact. Suppose that there is a point $(g, a) \in S E_{\theta}$. Then there is a net $\left(h_{\alpha},\left(g_{\alpha}, a_{\alpha}\right)\right)$ in $H \times(G \times A)$ such that $\left(g_{\alpha} h_{\alpha}^{-1}, h_{\alpha} a_{\alpha}\right) \rightarrow(g, a)$. Since $H$ is a quasi-compact, then $h_{\alpha} \rightarrow h$ for some $h$, and so $g_{\alpha} \rightarrow g h$ and $a_{\alpha} \rightarrow h^{-1} a$. Since 
$h\left(g h, h^{-1} a\right)=(g, a)$, then $\left(g h, h^{-1} a\right) \in \theta^{-1}(g, a) \cap \operatorname{cls}\left(\left(h_{\alpha},\left(g_{\alpha}, a_{\alpha}\right)\right)\right)=\emptyset$ which is a contradiction. Therefore, $S E_{\theta}=\varnothing$ and $\theta$ is proper.

4.1.6 Corollary: The map $\theta$ is closed.

4.1.7 Proposition. Let $G$ be a topological group and $H$ be a closed subgroup of $G$. Then for every $H$-space $A$ the action of $H$ on $G \times A$ is $w$-Cartan. Moreover, if $G$ and $A$ are $T_{2}$, then the action is also $w$-proper ( $G \times{ }_{H} A$ is Hausdorff).

Proof. We shall prove that $J(g, a)=\emptyset$ for every $(g, a)$. Suppose that $\left(g_{1}, a_{1}\right) \in$ $J(g, a)$ for some $\left(g_{1}, a_{1}\right) \in G \times A$. Then there are nets $h_{\alpha} \rightarrow \infty$ in $H$ and $\left(g_{\alpha}, a_{\alpha}\right) \rightarrow(g, a)$ such that $\left(g_{\alpha} h_{\alpha}^{-1}, h_{\alpha} a_{\alpha}\right) \rightarrow\left(g_{1}, a_{1}\right)$. Then $g_{\alpha} h_{\alpha}^{-1} \rightarrow$ $g_{1}$ and $h_{\alpha}^{-1} \rightarrow g^{-1} g_{1}$. Since $H$ is a closed, we have a contradiction. Therefore, $J(g, a)=\varnothing$. If $G \times A$ is $T_{2}$, then $\theta$ is $w$-proper.

4.1.8 Proposition. Let $K$ be a quasi-compact subset of $T_{2}$ topological group $G$, and $H$ be a closed subgroup of $G$. Then for a $T_{2} H$-space $A$, the restriction of quotient map $q: K \times A \rightarrow G \times{ }_{H} A$ is proper.

Proof. To show that $E_{q}=\emptyset$, suppose that there is a point $[g, a] \in E_{q}$. Then there is a net $\left(k_{\alpha}, a_{\alpha}\right) \rightarrow \infty$ in $K \times A$ such that $\left[k_{\alpha}, a_{\alpha}\right] \rightarrow[g, a]$. We have $k_{\alpha} \rightarrow g \wedge$ $a_{\alpha} \rightarrow a$ which contradicts our assumption. Therefore, $E_{q}=\emptyset$. Since $K \times A$ is $T_{2}, q$ is proper.

4.1.9 Corollary. The map $A \rightarrow[e, A]$ is a $G$ - map and an embedding from $A$ into the orbit space $G \times_{H} A$.

Proof: It is a restriction of the quotient map $q$ on $\{e\} \times A$ which is proper (closed) by proposition (4.1.8).

\subsubsection{Remark.}


1. Since $G \times{ }_{H} S$ is considered a $G$-space by the formula $g\left[g^{\prime}, s\right]=\left[g g^{\prime}, s\right]$, then every $G$-orbit $[g, s]$ can be written as $G[e, s]$ where $e$ is the identity element.

2. If $s \in S$ and $g \in G$, then $G_{[g, s]}=H_{s}$. If $g \in G_{[e, s]}$ and $g[e, s]=[e, s]$, then $[g e, s]=[g, s]=[e, s]$ this implies to that there exists an $h \in H$ such that $g=e h^{-1}$ and $h s=s$. Thus $g=h$ and $g \in H_{s}$. On the other hand, if $h \in$ $H_{s}$, then $h[e, s]=[h, s]=\left[h h^{-1}, h s\right]=[e, s]$. Therefore, $G_{[g, s]}=H_{s}$

3. If $g_{1}, g_{2} \in G$ and $s_{1}, s_{2} \in S$, then the set $\left(\left(\left[g_{1}, s_{1}\right],\left[g_{2}, s_{2}\right]\right)\right)=$ $g_{2}\left(\left(\left\{s_{1}\right\},\left\{s_{2}\right\}\right)\right)_{H} g_{1}^{-1}$. Take $g \in\left(\left(\left[g_{1}, s_{1}\right],\left[g_{2}, s_{2}\right]\right)\right)$, then $\left[g g_{1}, s_{1}\right]=$ $\left[g_{2}, s_{2}\right]$. By the definition of action, there is an $h \in H$ such that $g g_{1} h^{-1}=$ $g_{2}$ and $h s_{1}=s_{2}$. Thus, $h \in\left(\left(\left\{s_{1}\right\},\left\{s_{2}\right\}\right)\right)_{H}$ and $g=g_{2} h g_{1}^{-1} \in$ $g_{2}\left(\left(\left\{s_{1}\right\},\left\{s_{2}\right\}\right)\right)_{H} g_{1}^{-1}$. Conversely; if $g \in g_{2}\left(\left(\left\{s_{1}\right\},\left\{s_{2}\right\}\right)\right)_{H} g_{1}^{-1}$, then $g=$ $g_{2} h g_{1}^{-1}$ for some $h \in\left(\left(\left\{s_{1}\right\},\left\{s_{2}\right\}\right)\right)_{H}$. Since $h s_{1}=s_{2}$, then we have $g\left(g_{1} h^{-1}\right)=g_{2}$ or $\left(g g_{1}\right) h^{-1}=g_{2}$. Therefore, $\left[g g_{1}, s_{1}\right]=g\left[g_{1}, s_{1}\right]=$ $\left[g_{2}, s_{2}\right]$ and $g \in\left(\left(\left[g_{1}, s_{1}\right],\left[g_{2}, s_{2}\right]\right)\right)$.

\subsection{Slice in $w$-Actions}

In this section, we shall follow the definition of Biller for slice. However, we will not consider separation axiom properties on $G$ and $X$.

4.2.1 Definition. Let $G$ be a topological group that acts continuously on a space $X$ and let $H$ be a closed subgroup of $G$. An $H$-slice in $X$ for the action of $G$ is an $H$ invariant subset $S \subseteq X$ such that the continuous $G$-invariant map $G \times_{H} S \rightarrow X$ which is given by $[g, s] \mapsto g s$ is an open embedding. Moreover, the action said to 
be having enough slice if for each point $x \in X$, there is a closed quasi-compact subgroup $K$ of $G$ such that $x$ is contained in a $K$-slice.

4.2.2 Remark. Taking the definition (4.2.1), it is obvious that GS is open in $X$. Consider a map $G S \rightarrow G / H$ which takes $g s \mapsto g H$. It is well-defined since if $g_{1} s_{1}=g_{2} s_{2}$, then $g_{2}^{-1} g_{1} s_{1}=s_{2}$ and because $S$ is only invariant under $H$, then $g_{2}^{-1} g_{1} \in H$ and $g_{1} H=g_{2} H$. It is clear the map is an equivariant and a surjection. Its continuity comes from the continuity of the map $G \times{ }_{H} S \rightarrow G / H$ and the openness and surjection of the map $G \times_{H} S \rightarrow G S$. We have that the definition (4.2.1) implies the Palais' (definition 4.1.1). Therefore, in the Palais setting the two definitions are equivalent (lemma 4.1.4).

The following result comes already from our sense of $w$-Cartan action.

4.2.3 Proposition. If $G$ is a topological group and $S$ is an $H$-space where $H$ is a subgroup of $G$, then the action of $G$ on $G \times_{H} S$ is $w$-Cartan if and only if the action of $H$ on $S$ is $w$-Cartan.

Proof: Assume that $S$ is a Cartan $H$-space. Suppose there is $s \in S$ and $g \in G$ such that $[g, s] \in J([g, s])$. Then then there is a net $g_{\alpha}^{\prime} \rightarrow \infty$ in $G$ and $\left[g_{\alpha}, s_{\alpha}\right] \rightarrow[g, s]$ such that $g_{\alpha}^{\prime}\left[g_{\alpha}, s_{\alpha}\right]=\left[g_{\alpha}^{\prime} g_{\alpha}, s_{\alpha}\right] \rightarrow[g, s]$. This implies that $g_{\alpha} \rightarrow g$ and $g_{\alpha}^{\prime} g_{\alpha} \rightarrow g$ and $s_{\alpha} \rightarrow s$. By the definition of the twisted product, let $h_{\alpha} \in H$ be a net in $H$ such that $g_{\alpha}^{\prime} g_{\alpha} h_{\alpha}^{-1} \rightarrow g$ and $h_{\alpha} s_{\alpha} . \rightarrow s$. Since $s \notin J(s)$, then we have $h_{\alpha} \rightarrow h \in H$. Thus $g_{\alpha}^{\prime} \rightarrow g h g^{-1} \in G$ which is a contradiction. Conversely, suppose that there is a point $s \in S$ such that $s \in J(s)$. Then there is a net $h_{\alpha} \rightarrow \infty$ in $H$ and a net $s_{\alpha} \rightarrow s$ in $S$ with $h_{\alpha} s_{\alpha} \rightarrow s$. Since the map $G \times S \rightarrow G \times{ }_{H} S$ is continuous and $\left(e, h_{\alpha} s_{\alpha}\right)$ is a net in $G \times S$ converges to $(e, s)$, then $\left[h_{\alpha}, s_{\alpha}\right]=$ $h_{\alpha}\left[e, s_{\alpha}\right]=\left[e, h_{\alpha} s_{\alpha}\right] \rightarrow[e, s]$ in $G \times_{H} S$ this means that $[e, s] \in J([e, s])$ which contradicts that $G \times_{H} S$ is $w$-Cartan. Therefore, the proposition is true. 
4.2.4 Proposition. Let $G$ be a topological group, and $H$ be a closed subgroup of $G$ which acts on $H$. Then there is a homeomorphism $\bar{\mu}$ from $S / H \rightarrow\left(G \times{ }_{H} S\right) / G$. Proof. It follows from the composition of induced maps from composition maps $\mu$ such that the following diagram is commutative,

$$
\begin{array}{ccccc}
\mu: H \times S & \rightarrow & G \times S & \rightarrow & G \times \times_{H} S \\
\downarrow & & \downarrow & & \downarrow \\
\bar{\mu}: S / H \cong H \times{ }_{H} S & \rightarrow & G \times \times_{H} S \rightarrow & \left(G \times \times_{H} S\right) / G
\end{array}
$$

and is determined by the maps $(h, s) \rightarrow(h, s) \rightarrow[e, s]$, and then $\bar{\mu}(H s)=G[e, s]$ which is a continuous bijection. To show $\bar{\mu}$ is open, take $U S / H$ and open $S / H$, then its image under $\bar{\mu}$ is exactly the image of the subset $G \times U$ under $q \circ \mu$ which is open where $q$ is the canonical quotient map.

4.2.5 Corollary. If $G$ is a topological group and $S$ is an $H$-space where $H$ is a subgroup of $G$. Then the action of $G$ on $G \times_{H} S$ is $w$-proper ( $w$-strongly proper) if and only if the action of $H$ on $S$ is $w$-proper ( $w$-strongly proper).

Proof. It follows immediately from the previous proposition.

4.2.6 Proposition. If $X$ is a $T_{2} G$-space and $X$ has enough slice, then $X$ is $w$-Cartan. Proof. Assume that the action has enough slice. To show that for every point $x \in$ $X$ the condition $x \notin J(x)$, pick a point $x \in X$. Then there is a closed quasi-compact subgroup $H$ such that $x$ contained in $H$-slice $S \subseteq X$. That is, the map $\rho: G \times_{H} S \mapsto$ $X$ is an open embedding. Since $H$ is quasi-compact and $S$ is an $H$-space, then the action of $H$ on $S$ is $w$-Cartan, that is $x \notin J(x)$ with respect to this $H$-space $S$. By proposition (4.2.3), the $G$-action on $G \times{ }_{H} S$ is $w$-Cartan. Since the map $\rho$ : $[g, s] \rightarrow g s$ is an open embedding, then $g x \notin J(g x)$ and hence $x \notin J(x)$ in the $G$ space $G S$ which is an open subset of $X$. Therefore, $x \notin J(x)$ in the $G$-space $X$ and the action of $G$ on $X$ is $w$-Cartan. 
4.2.7 Remark. For the slice theorem in the Palais setting we know that $w$-notions and Palais' notions coincide. We may also then give the inverse of the other direction of the above proposition, but we need the following facts.

4.2.8 Definition. A locally quasi-compact group is called almost connected provided that the orbit space $G / G_{\circ}$ is quasi-compact (and hence compact since every totally disconnected topological group is $T_{2}$ ).

4.2.9 Lemma 1. If $G$ is locally quasi-compact, and $H$ is a closed subgroup of $G$, then $H$ is locally quasi-compact and the orbit space $G / H$ is locally compact. Proof. It is obvious.

4.2.10 Lemma. [23] If $G$ is locally quasi-compact, then $G / G_{\circ}$ contains arbitrarily small compact open subgroups and corresponding to these groups, $G$ contains open subgroups $G^{\prime}$ such that $G^{\prime} / G_{\text {o }}$ is compact.

4.2.11 Remark. According to lemma 2, every locally compact group contains an arbitrary open subgroup which is almost connected.

4.2.12 Lemma. [15] If the component $G_{\text {o }}$ of the identity element of a locally compact group has a compact factor group $G / G_{0}$, then every neighborhood of the identity of $G$ contains a compact normal subgroup $N$ such that $G / N$ is a Lie group.

4.2.13 Lemma. [9] Let $G$ be a locally compact group acting properly on a completely regular space $X$. If $x \in X$ and $N$ is a compact normal subgroup of $G$ such that $G / N$ is a Lie group, then $x$ is contained in a $G_{x} N$-slice for the action of $G$ on $X$.

4.2.14 Theorem. An action of a locally compact group $K$ on a completely regular space $X$ has enough slice if and only if it is $w$-Cartan.

Proof. The first direction of the proof comes immediately from the proposition (4.2.6). Assume that an action of a locally compact group $K$ on $X$ is $w$-Cartan. Since $K$ is a locally compact, then $K$ contains an arbitrary open almost connected 
subgroup $K^{\prime}$ by lemma (4.2.10) such that $G=K^{\prime} / G_{\circ}$ is compact. By lemma (4.2.12), every neighborhood of the identity element $e$ in $K^{\prime}$ contains a compact normal subgroup $N$ such that $K^{\prime} / N$ is a Lie group. Since $X$ is a $w$-Cartan $G$-space, every point $x \in X$ has a thin (open) neighborhood $U$ on which $G$ acts $w$-Palais proper. Then by lemma (4.2.13), $x$ contained in $K_{x}^{\prime} N$-slice $S$ with respect to the action of $G$ on $X$.

According to our aim, namely putting the three actions in clear arrangement, we return to Baum-Connes-Higson proper, definition (3.1.1). We shall show the latter proper is just strongly proper under its assumption. The three conditions of the definition (3.1.1) are equivalent to that $X$ is $w$-Cartan.

4.2.15 Proposition. Under the assumption of the definition (3.1.1), the three conditions of the definition are equivalent to that $X$ is $w$-Cartan.

Proof. Assume the three conditions of the definition (3.1.1) are satisfied. By proposition (3.2.11), it suffices to show every point has a thin neighborhood. Take a point $x \in X$, there is a triple $(U, H, \rho)$ such that the three conditions are satisfied. Put $S=\rho^{-1}(H)$, and $\rho\left(g^{-1} x\right)=H$ for some $g \in G$. Then $S$ is an $H$-invariant closed subset of $U$. Since $G$ is a locally compact and $H$ is compact, then there is a compact neighborhood $\bar{V}$ of $H$ in $G / H$. Let $W=\cup\{\bar{v}: \bar{v} \in \bar{V}\}$. Then $W$ is a compact neighborhood $H$ in $G . V=\rho^{-1}(\bar{V})$ is a neighborhood of $S$ in $U$. It is easy to see that $g^{-1} x \in S \subseteq V$ and $((V, V))=W$, that is $g^{-1} x$ has a thin neighborhood $V$ in $U$ that is equivalently $x$ has a thin neighborhood $g V$ in $U$ and hence it is open in $X$. Therefore, $X$ is $w$-Cartan. Conversely, by theorem (4.2.14) if $x \in X$, then $x$ is contained in $H$-slice, say $S$. Put $(G S, H, \rho)$ where $\rho(g v)=g H$ for every $g \in G$ and $v \in V$. The triple $(G S, H, \rho)$ satisfies the three conditions of the definition (3.1.1) 
4.2.16 Corollary. Let $G$ be a locally compact and second countable ( $T_{2}$ included) and let $G$ act continuously on a metrizable space $X$. Then the following are equivalent.

1. $X$ is a $W$-strongly proper $G$-space.

2. $X$ is a $B a-C o-H i$ proper $G$ - space.

Proof. $(2 \rightarrow 1)$ follows from proposition (4.2.15), $X$ is paracompact and hence $X / G$ is as well. For $(1 \rightarrow 2)$, it is immediately implied by proposition (4.2.15).

4.2 17 Remark. Palais has presented many results using the slice theorem. Here we also confirm some of the results in the context of an arbitrary locally compact (non-necessary Lie) group.

4.2.18 Corollary. Let $S$ be $H$-slice at a point $x$ in $X$. Then there is a neighborhood $V$ of $x$ such that $y \in V$ implies $G_{y}$ is conjugate in $G$ to a subgroup of $H$. Moreover if $O$ is any neighborhood of $e \in G$, then $V$ can be chosen so that in fact for each $y \in V$ we have $G_{y} \subseteq g H^{-1}$ for some $g \in O$.

Proof. Take $x \in X$ and $S$ be a $H$-slice containing $x$. Then there is a $G$-map $f: G S \rightarrow$ $G / H$. Take an open neighborhood of the identity element $e$ in $G$, say $N$. Put $U=$ $f^{-1}(N H / H)$, then $U$ is an open neighborhood of $x$ in $G S$ and hence in $X$. For every $u=n h$ in $U$, then we have $G_{u} \subseteq G_{f(u)}=G_{n H}=n H n^{-1}$.

4.2.19 Corollary. Let $S$ be an $H$-slice at a point $x$ in $X$. If $K$ is any closed subgroup of $G$ which includes $H$, then $K S$ is a $K$-slice in $X$ containing $x$.

Proof. Let $f: G \times_{H} S \rightarrow G / H$ be the $G$-map that associated with the $H$-slice $S$. Since $H<G$, there is a canonical map $g: G / H \rightarrow G / K$ given by $g H \rightarrow g K$. The composition of the two is a map $G \times_{H} S \rightarrow G / K$. Since $G \times_{K}\left(K \times_{H} S\right) \cong G \times_{H} S$ and $\left(K \times_{H} S\right) \cong K S$ since $S$ is $H$-slice under the subgroup $K$. Therefore, the 
composition is the $G$-map $G \times_{K} K S \rightarrow G / K$ and hence $K S$ is $K$-slice containing $x$ 


\section{Bibliography}

[1] Al-Sarray M. J.: Convergence characterization of proper mapping Journal

Of science college, Salahuddin University /Erbil the first Iraqi- French

Mathematics Conference, Special Issue- Vol.22 -2010.

[2] Al-Sarray M. J.: Exceptional sets as characteristic for closed, compact and proper functions, Journal of basic education. No (60), (2012).

[3] Al-Sarray M. J.: On Strongly Proper Actions, M.Sc. Thesis, College of Science, University of AL-Mustansiryah, 2000.

[4] Abels, H.: A universal proper G-space, Math. Z. 159 (1978), 143-158.

[5] Antonyan S.A.: Proper actions on topological groups: Applications to quotient spaces, Proc. Amer. Math. Soc. 138 (10) (2010) 3707-3716.

[6] Baum P., Connes A. and Higson N.: Classifying space for proper actions and $K$ - theory of group $C^{*}$-algebras. $C^{*}$-algebras: 1943-1993

(San Antonio, TX, 1993), Amer. Math. Soc., Providence, RI (1994), pp. 240-291.

[7] Bhatia N. P., and Szego G. P.: Stability Theory of Dynamical Systems, Springer-Verlag, 1970.

[8] Biller H., Characterizations of proper actions, Preprint 2211, Fachbereich Mathematik, Technische Universit" at Darmstadt, 2002, http://wwwbib.mathematik.tu-darmstadt.de/ Math Net/Preprints/Listen/shadow/pp2211.html; to appear in Math. Proc. Cambridge Philosophical Society.

[9] Biller H.: Proper actions on cohomology manifolds. Trans. Amer. Math. Soc. 335 (2003), 407-432.

[10] Biller H.: Actions of compact groups on spheres and on generalized quadrangles, Dissertation, Stuttgart, 1999, http://elib.unistuttgart.de/opus/volltexte/1999/566.

[11] Bourbaki N., General Topology, Chapters 1-4, Addison-Wesley, 1966.

[12] Bredon, G. E., Introduction to Compact Transformation Groups, Academic Press, 1972.

[13] Dydo W.: Proper G - spaces, J. Diff. Geometry, 9:565-569(1974).

[14] Engelking R.: General Topology, 2nd ed. Sigma Series in Pure Mathematics 6 (Heldermann Verlag, 1989). 
[15] Gluskov V. M.: The structure of locally compact groups and Hilbert's fifth problem. Amer. Math. Soc. Transl., Ser. 2, 15 (1960), 55-93, transl. of Usp. Mat. Nauk. 12 (1957), no. 2, 3-41.

[16] Gotô, M., Yamabe H.: On some properties of locally compact groups with no small subgroup. Nagoya Math. J. 2 (1951), 2 https://projecteuclid.org/euclid.nmj/1118764736.

[17] Hofmann K. H.: Introduction to Topological Groups, Jour, 2005.

[18] Higgins, P.J.: An Introduction to Topological Groups, London Mathematics Society Lecture Notes Series 15, 1974.

[19] Kaplansky I.: Lie algebras and locally compact groups. Chicago Lectures in Mathematics (The University of Chicago Press, 1971).

[20] Karppinen S.: The existence of slices in G-spaces, when G is a Lie group, Department of Mathematics and Statistics University of Helsinki, 2016.

[21] Koszul J. L.: Lectures on Groups of Transformations (Tata Institute of Fundamental Research, Bombay, 1965).

[22] Kawakubo, K.: The Theory of Transformation Groups, Oxford University Press, 1991.

[23] Montgomery, D. and Zippin, L.: A theorem on Lie groups, Bull. Amer. Math, Soc., 48(1942), 448-452.

[24] Munkres J. M.: Topology, 2nd ed., Prentice-Hall, Upper Saddle River, NJ, 2000.

[25] Seymour Lipschutz: Theory and Problems of General Topology, Schaum's Outline Series in Mathematics, Megraw-Hill Company, 1965.

[26] Stranzalos, P.: Action by Isometries, Transformation groups, proceedings of a conference, Osaka, Japan, Dec: 16-21: 319-325(1987).

[27] Palais, R. S.: On the existence of slices for actions of non-compact Lie groups, Ann. of Math. 73 (1961) 295-323.

[28] Palais, R. S.: Classification of G-spaces, Mem. Amer. Math. Soc., No. 36, 1960.

[29] Palais, Borel, et al. Seminar on Transformation Groups. (AM-46). Princeton University Press, 1960.

[30] WILLARD, S.: General Topology, Addison-Wesley publishing company, Inc,1970. 